\title{
Probing strongly interacting W's at the ILC with polarized beams
}

\section{B. Ananthanarayan, ${ }^{a}$ Monalisa Patra ${ }^{a}$ and P. Poulose ${ }^{b, c}$}

\author{
${ }^{a}$ Centre for High Energy Physics, Indian Institute of Science, \\ Bangalore 560 012, India \\ ${ }^{b}$ DESY FLC, \\ Notkestrasse 85, Hamburg 22607, Germany \\ ${ }^{c}$ Department of Physics, Indian Institute of Technology Guwahati, \\ Guwahati 781 039, India ${ }^{1}$ \\ E-mail: anant@cts.iisc.ernet.in, monalisa@cts.iisc.ernet.in, \\ poulose@itt.ernet.in
}

\begin{abstract}
We study the possibility of fingerprinting a strongly interacting $W$ boson sector which is consistent with present day LHC searches at the ILC with longitudinal as well as transversely polarized electron and positron beams. We account for the final state interaction using a suitable Omnès formalism in terms of a plausible resonance description, and carry out thorough analyses of cross sections, asymmetries and angular distributions of the $W^{\prime} s$. We carry out a comparison with other extensions of the Standard Model, where heavy additional $Z^{\prime}$ bosons arise naturally. We also consider the effect of the strong final state interaction on a correlation that depends on $\left(\phi_{-}-\phi_{+}\right)$, where the $\phi_{\mp}$ are the azimuthal angles of decay leptons, and find that it is a useful discriminant.
\end{abstract}

KEYWORDS: e+-e- Experiments

ARXIV EPRINT: 1112.5020

\footnotetext{
${ }^{1}$ Permanent address.
} 


\section{Contents}

1 Introduction 1

2 Strong final state interaction in $W W$ channel 4

$\begin{array}{lll}3 & \text { Beam polarization } & 6\end{array}$

3.1 Longitudinal polarization 8

$\begin{array}{llr}3.2 & \text { Transverse polarization } & 8\end{array}$

4 Numerical analyses with $W^{\prime} s \quad 8$

4.1 Total cross section $\quad 9$

4.2 Angular distribution of $W_{L} W_{L}$ and $W_{L} W_{T} \quad 11$

$\begin{array}{lll}4.3 & \text { Forward backward asymmetry } & 13\end{array}$

$\begin{array}{lll}4.4 & \text { Left right (LR) asymmetry } & 15\end{array}$

$\begin{array}{lll}4.5 & \text { Azimuthal distribution of } W^{\prime} s & 16\end{array}$

$\begin{array}{llr}5 & \text { Inclusion of decays } & \mathbf{1 8}\end{array}$

$\begin{array}{ll}5.1 \text { Azimuthal distribution of the decay leptons } & 18\end{array}$

$\begin{array}{lll}5.2 & \text { Energy distribution of the decay leptons } & 19\end{array}$

6 Discussions and conclusions $\quad 20$

$\begin{array}{ll}\text { A Form factor parametrizations } & \mathbf{2 1}\end{array}$

A.1 Non-resonant SFI 21

$\begin{array}{lll}\text { A.2 Gounaris-Sakurai parametrization } & 22\end{array}$

$\begin{array}{lll}\text { A.3 } & \text { Breit-Wigner parametrization } & 22\end{array}$

\section{Introduction}

A strongly interacting $W$ bosons sector is now a definite possibility especially in the light of the present LHC results [1-8]. If there is no light Higgs, it could be either $(a)$ that it is very massive and could even violate the unitarity bound of $\sim 1.2 \mathrm{TeV}$, in which case there would be new physics to restore unitarity via strong gauge boson interaction in gauge boson scattering processes, or $(b)$ that there is no Higgs, which is possible if electroweak symmetry were to be broken dynamically through a Landau-Ginzburg type scenario due to the existence of an order parameter $\Phi$, leading again to strong final state interactions (SFI) of the weak gauge bosons. In slight variation to the strong gauge boson interactions without a Higgs boson, the possibility of a light Higgs boson along with a strongly interacting gauge sector has emerged in the recent past. In this scenario, there exists a light Higgs boson, whose dynamics are not strong enough to unitarize the gauge boson scattering. Unlike the case of the SM, the cross section still grows even after crossing the light Higgs resonance. 
In most of the phenomenological studies of scenarios without a light Higgs boson, the onset of strong interactions among the gauge bosons is viewed in a fashion very similar to that of the strong interaction dynamics of pions. While pions are considered as the pseudo Goldstone Bosons of chiral symmetry breaking in low energy QCD, the longitudinal modes of the weak gauge bosons are essentially the Goldstone modes of electroweak symmetry breaking (EWSB). This analogy, along with the equivalence theorem of weak gauge bosons, which states that at high energies (compared to their masses) the gauge boson scattering cross sections are essentially equal to the cross sections of the Goldstone Boson scattering [9-13]. It is possible to have some understanding of the gauge boson scattering in this strongly interacting regime: at the electroweak scale, a "chiral effective Lagrangian" [14-17] can be constructed along the lines of low-energy QCD, with different interaction terms among the gauge bosons with appropriate coefficients. These coefficients can in principle be computed using the underlying theory at high energies. Unlike in the case of pion interactions, where the underlying theory is QCD, such a theory is not known in the weak interaction case. In the absence of such a theory, these coefficients are considered as parameters to be fixed from experiments. One important feature of strong pion interactions is the existence of various resonances in their scattering. Therefore, adapting a similar scheme for the weak gauge boson scattering implies the existence of resonances beyond the unitarity violating scale. In the chiral effective Lagrangian approach, effect of such resonances at electroweak scale can be accommodated into the various coefficients of the Lagrangian by integrating out the heavy resonances. Early phenomenological studies of strong gauge boson, in the absence of a light Higgs, relevant to LHC are summarized in ref. [18-21]. Gauge boson scatterings in processes like $q q \rightarrow q q V V$, where $V=W, Z$ were computed within, what is known as, the equivalent gauge boson approximation (EGBA) [22-26]. In such an approximation the $V V$ scattering cross section is folded with the probability distribution of $W$ and $Z$ bosons contained in the proton. More recently various analyses have pointed out the inadequacy of the EGBA, suggesting that a full analysis of the process is necessary $[27,28]$ to take into account the non-negligible contributions coming from off-shell gauge bosons, as well as the possibility of the same final state arising through intermediate states other than those with $V$ radiation from protons. In particular in ref. [28] contributions of various different possible resonances in the $V V$ scattering within the chiral Lagrangian framework, have been included using the event generator called WHIZARD [29-31] to study the phenomenology at LHC.

Returning now to a light Higgs $\sim 115-130 \mathrm{GeV}$, two distinct cases relevant in this context are $(a)$ with additional Higgs bosons, which restore unitarity [32], and (b) without any other Higgs bosons in the spectrum, in which case unitarity is restored by new physics [33]. In these cases, gauge bosons become strongly interacting after crossing the light Higgs resonance. Thus, even with the discovery of a light scalar particle in the small window available at the LHC $[7,8]$, a detailed analysis of the gauge boson interactions, especially probing the signatures of their strong interactions in processes with $W$ and/or $Z$ bosons in the final state is essential to understand the mechanism of EWSB, as emphasised by $[34,35]$. Phenomenology of such models at LHC are considered in ref. [32, 33, 36, 37]. A comprehensive review of various models and approaches to study the strong gauge boson interactions may be found in [38]. 
In the effective phenomenological approach, the underlying theoretical origin of these resonances is not addressed. The ultraviolet completion of such effective theories are expected to explain the origin of such resonances. In most cases, it is argued [28, 34], that the vector bosons are the dominant resonances. Among the renomarlizable theories explaining the origin of vector resonances, the idea of dynamical EWSB of technicolour models [39] proposing a composite scalar sector with a techni- $\rho$ meson playing the role of the vector resonance mentioned above has been studied in detail in the past decades. While aesthetically very pleasing, in their original form, these models fail to comply with electroweak precision measurements. In a philosophically different approach, the BESS (Breaking Electroweak Symmetry Strongly) models [40-44] consider the $\rho$ resonance as a gauge boson of a hidden $\mathrm{SU}(2)$, introducing strong interactions in the weak gauge boson sector. This model, again, is ruled out by precision electroweak measurements, unless the $\rho$ is fermiophobic. With the possibility of low energy gravity scenarios arising through large compactified extra space dimensions, an interesting set of models have emerged in the recent past. These Higgsless models [45-57] have been proposed with gauge theories in five dimensions. In such models the Kaluza-Klein towers of the gauge bosons act as the moderators of unitarity in gauge boson scattering in four dimensions. In a different perspective, in many versions, these models can be considered as four dimensional deconstructed theories with a chain of $\mathrm{SU}(2)$ gauge groups [58-75]. The BESS model above can be considered as some special case of the deconstructed models. A general discussion of relation between the composite models with and without gauge theory is presented in [76, 77]. The effect of such a vector resonance (in the absence of any other resonance) in $V V$ scattering was recently analyzed in the context of LHC [34, 78]. In these models as well, when the new gauge bosons couple to the SM fermions, evading precision constraints is difficult, but possible with some modifications [79, 80].

Coming to the leptonic colliders, similar to the case mentioned above in the context of LHC, $e^{+} e^{-} \rightarrow l l^{\prime} W^{+} W^{-}$, where $l, l^{\prime}=e, \mu, \nu_{e}, \nu_{\mu}$ have been investigated for strong $V V$ scattering. A large volume of phenomenological studies available in this case are summarized in refs. [81-84]. This process is sensitive to scalar and tensor resonances, as well as the vector resonances arising in gauge boson scattering. On the other hand, the process $e^{+} e^{-} \rightarrow W^{+} W^{-}$, which we consider in the present work, has the advantage that only vector resonances are involved. The fact that the cross section (at ILC energies) for $e^{+} e^{-} \rightarrow W^{+} W^{-}$is about three orders of magnitude larger than that of $e^{+} e^{-} \rightarrow l l^{\prime} W^{+} W^{-}$ also helps the former. For reviews of phenomenological work on $e^{+} e^{-} \rightarrow W^{+} W^{-}$based on "chiral electroweak Lagrangian", see the work of Barklow $[85,86]$. Some early analyses of $e^{+} e^{-} \rightarrow W^{+} W^{-}$within the framework of the BESS model studied the contribution of the additional $\rho$ in the $s$-channel [87-89]. These were extended to include the decay spectrum of the $W$ 's with the leptonic energy and angle distributions acting as $W$ polarization analyzers [90]. These effects are almost negligible unless one is very close to the new vector resonance, owing to their highly constrained fermionic couplings. However, even in the absence of fermion couplings, these vector resonances can leave their impact through strong final state interactions (SFI) in the same process. In a model independent way, the effect of a single vector boson resonance such as this in the SFI can easily be parametrized by 
introducing suitable form factors in the $l=1$ partial wave amplitude of $e^{+} e^{-} \rightarrow W^{+} W^{-}$. With this philosophy, one may approximate the effect of the resonance through a GounarisSakurai (GS) form factor, as considered in [91], or even through a Breit-Wigner (BW) form factor, as considered in [92]. An improved treatment of vector boson resonance is to introduce a suitable Omnès function. Ref. [93] has used the Omnès function with Padé unitarization method considered to implement the phase shift of the $P$ partial wave in $e^{+} e^{-} \rightarrow W^{+} W^{-}$. Studies in $W$ pair production at a $\gamma \gamma$ collider is considered in ref. [94], where again the SFI is modeled through a spin-2 resonance, the effect of which is considered through a BW form factor introduced in the $l=2$ partial wave.

Revisiting the $W$ pair production at ILC, in the present work, we study the effect of such a $\rho$ resonance in this process. While inspired by the theoretical scenarios described earlier, we consider a model independent approach in our study. Here, we will follow the treatment adopted in refs. [91-93], with suitably defined Omnès function to be described. In an earlier analysis of the same process [95], we had considered the effect of the presence of heavy $Z^{\prime}$ boson, arising in many Grand Unified scenarios including the $E_{6}(\chi, \psi, \eta)$, Left Right Symmetric Model (LRSM) and the Alternate Left Right Symmetric Model (ALRSM) [96], as well as in the Little Higgs Models (LHM) [97]. With the effect of SFI with vector resonance expected to mimic the presence of a $Z^{\prime}$ in the $s$-channel, it is prudent to distinguish between these. The role of beam polarization at the ILC in probing new physics and disentangling various possible scenarios have been demonstrated through many examples (for example, see [98] for a recent review). In this work we exploit the potential of beam polarizations, both longitudinal polarizations (LP) and the transverse beam polarization (TP) expected to be available at ILC to study the new physics effects. Note that the possibilities with TP have not received any attention in studies involving SFI, although studies on effects of anomalous couplings in case of $W$ pair production at linear colliders are considered by refs. [99-102].

The plan of this article is the following. In section 2 we very briefly review the formalism to introduce the SFI through a modification of the $l=1$ partial wave with the Omnès function as applied to the process under consideration. In section 3 we categorise the different type of polarizations considered in our analyses. In section 4 we discuss different observables sensitive to the new effect, and present our numerical results, where a comparison with the effect of $Z^{\prime}$ in the same process is considered. In this section we also attempt to discriminate between the two effects with the help of various observables. In section 5, we probe the effects, through the azimuthal distribution of the decay leptons, and the single energy distribution. In section 6 we provide discussions and conclusions. In appendix A we present some details of the parametrizations used in section 2.

\section{Strong final state interaction in $W W$ channel}

The process $e^{+} e^{-} \rightarrow W^{+} W^{-}$in the SM proceeds through the $s$-channel exchange of $\gamma$ and $Z$, and a $t$-channel $\nu$ exchange, as shown in figure 1 .

In this work since we are interested in SFI arising in the EWSB sector, only the amplitudes involving longitudinal $W$ 's (denoted as $W_{L}$ henceforth) are modified, leaving 

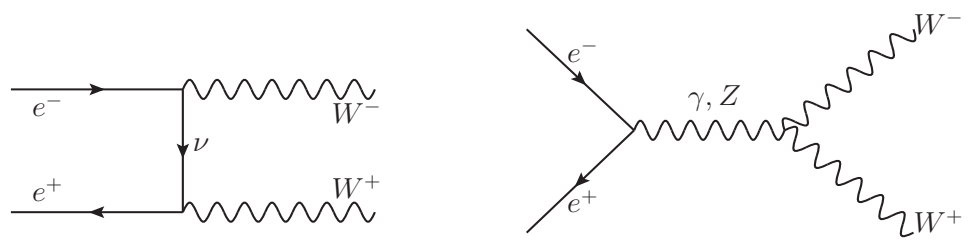

Figure 1. Feynman diagrams contributing to the process $e^{+} e^{-} \rightarrow W^{+} W^{-}$in the SM.

the helicity amplitudes involving transverse $W$ 's unaffected. The strong interaction through vector $\rho$ resonance affects only the $l=1$ partial wave. Thus, representing the SFI through an Omnès function $\Omega(s)$, the invariant amplitudes involving $W_{L}$ are modified. Noting that the $\gamma$ and $Z$ exchange $s$-channel contributions are pure $P$ waves, whereas the $\nu$ exchange $t$-channel has all partial waves with $l \geq 1$, the amplitude corresponding to $s$-channel of the $W_{L} W_{L}$ production is modified as:

$$
M_{L L}^{\gamma+Z}(s, \theta) \rightarrow \Omega(s) M_{L L}^{\gamma+Z}(s, \theta) .
$$

The effect of the SFI in the $t$-channel can be presented in the modification of the $l=1$ partial wave amplitude, leaving the other partial waves unaffected. Schematically therefore

$$
M_{L L}^{\nu, l}(s, \theta) \rightarrow \Omega(s) M_{L L}^{\nu, l=1}(s, \theta)+M_{L L}^{\nu, l>1}(s, \theta) .
$$

We have to isolate the $l=1$ partial wave, with the helicity amplitude for $W_{L} W_{L}$ defined in terms of partial waves as, ${ }^{1}$

$$
M_{L L}^{\nu, l=1}(s, \theta)=\frac{3}{4 \pi} d_{m, 0}^{1 *}(\theta) P_{L}^{1}(s), \quad m= \pm 1
$$

where

$$
d_{m, 0}^{1}(\theta)=-\frac{1}{\sqrt{2}} \sin \theta
$$

is the relevant rotation function. The $l=1$ partial wave is isolated by the projection,

$$
P_{L}^{1}(s)=2 \pi \int_{-1}^{+1} d(\cos \theta) d_{m, 0}^{1}(\theta) M_{L L}^{\nu, l}(s, \theta)
$$

The rest of the amplitude with $l>1$ is obtained by noting,

$$
M_{L L}^{\nu, l>1}(s, \theta)=M_{L L}^{\nu, l}(s, \theta)-M_{L L}^{\nu, l=1}(s, \theta) .
$$

We can rewrite eq. (2.2) as:

$$
M_{L L}^{\nu, l}(s, \theta) \rightarrow M_{L L}^{\nu, l}(s, \theta)+(\Omega(s)-1) M_{L L}^{\nu, l=1}(s, \theta) .
$$

\footnotetext{
${ }^{1}$ Note, that there has been an error in the formalism presented in ref. [92], which has also unfortunately trickled into the numerical effects. In eq. (12) of ref. [92] with the choice for projection made therein, a factor of $\sin \theta$ is missing in the second term, on the right hand side. Whereas in ref. [91] it was apparent that there are no significant effects in the cross section at centre of mass energies far away from the mass region of the resonance, the contrary finding was reported in ref. [92] which showed a significant deviation from the situation when there are no SFI already at a c.m. energy of $500 \mathrm{GeV}$. This is traced to the error noted here.
} 
The Omnès function, $\Omega(s)$ describes the effect of the vector resonance arising in the strong interaction of the final state $W$ 's. To describe this function, we consider a simple parametrization of the resonance through its mass, $M_{\rho}$ and its width, $\Gamma_{\rho}$. We chose the mass to vary from the unitarity limit $1.2 \mathrm{TeV}$ to $2 \mathrm{TeV}$, as the effect of a resonance with mass beyond a few $\mathrm{TeV}$ is expected to be small at an ILC running up to centre of mass (c.m.) energy of $1 \mathrm{TeV}$. For the choice of width, we rely on the constraints employed by the low energy chiral QCD on $\Gamma_{\rho}$. Accordingly we consider the relation

$$
\Gamma_{\rho}=\frac{m_{\rho}^{3}}{96 \pi v^{2}},
$$

where $v=246 \mathrm{GeV}$ is the Higgs vacuum expectation value in the SM. While adopting this choice for most of our analyses, we study the effect of varying $\Gamma_{\rho}$, considered as a free parameter, for certain observables in the next section. For one choice of $M_{\rho}=1200 \mathrm{GeV}$, we vary $\Gamma_{\rho}$ in the range $(50-140) \mathrm{GeV}$, to study the effects of such flexibility on one of the observables.

Given $M_{\rho}$ and $\Gamma_{\rho}$ the phase of the form factor $\delta$ may be obtained either from a Kmatrix, or from GS or BW parametrization as explicitly given in appendix A. Given $\delta$ we obtain the Omnès function,

$$
\Omega(s)=\exp \left[\frac{s}{\pi} \int_{4 m_{W}^{2}}^{\infty} \frac{\delta\left(s^{\prime}\right) d s^{\prime}}{s^{\prime}\left(s^{\prime}-s\right)}\right]
$$

Note that the form factor itself for a single channel elastic scattering is related to $\Omega(s)$ by $[103]$

$$
F(s)=\left(1+\frac{s}{a}+\frac{s^{2}}{b^{2}}+\cdots\right) \Omega(s)
$$

The constants $a, b, \cdots$ etc. are fixed from additional inputs from the underlying theory, or experiments, which we assume to be large. Since the correct prescription is the use of the Omnès function, for purposes of comparison we study the effect of replacing $\Omega(s) \rightarrow F(s)$ in some instances in the next section. We treat GS and BW parametrizations as low energy representation of the form factor only to generate $\delta$. However to contrast these effects we tabulate explicitly the values due to GS and BW parametrizations, and the corresponding Omnès function for the energies of interest given $M_{\rho}$ (and $\Gamma_{\rho}$ ). This is shown in table 1 , where we give the relevant Omnès function corresponding to the form factors used, for typical ILC energies of $\sqrt{s}=500,800$ and $1000 \mathrm{GeV}$.

\section{Beam polarization}

The use of beam polarization at ILC will significantly benefit the physics program as it is very useful in searches for new physics with small deviations from SM cross sections in two ways. Firstly, in many cases, suitably chosen beam polarization combinations can enhance the signal, and suppress the background. Secondly, it is possible to construct clever observables incorporating the beam polarization information. At the ILC, a beam polarization (both transverse as well as longitudinal) of $\geq 80 \%$ for electrons and $\geq 30 \%$ 


\begin{tabular}{|c|c|l|c|c|c|c|}
\hline $\begin{array}{c}M_{\rho} \\
(\mathrm{GeV})\end{array}$ & $\begin{array}{c}\Gamma_{\rho}(\mathrm{GeV}) \\
\text { eq. }(2.8)\end{array}$ & $\begin{array}{c}\sqrt{s} \\
(\mathrm{GeV})\end{array}$ & $\begin{array}{c}\text { GS } \\
\text { eq. (A.2) }\end{array}$ & $\begin{array}{c}\text { Omnès function } \\
\text { from GS }\end{array}$ & $\begin{array}{c}\text { BW } \\
\text { eq. (A.8) }\end{array}$ & $\begin{array}{c}\text { Omnès function } \\
\text { from BW }\end{array}$ \\
\hline \multirow{3}{*}{1200} & \multirow{3}{*}{95} & 500 & $3.849+i 0.055$ & $1.223+i 0.017$ & $0.993+i 0.083$ & $1.198+i 0.099$ \\
& & 800 & $5.750+i 0.347$ & $1.827+i 0.110$ & $0.982+i 0.135$ & $1.727+i 0.237$ \\
& 1000 & $10.225+i 1.803$ & $3.248+i 0.573$ & $0.939+i 0.240$ & $2.979+i 0.760$ \\
\hline \multirow{3}{*}{1600} & \multirow{2}{*}{224} & 500 & $4.599+i 0.058$ & $1.122+i 0.014$ & $0.982+i 0.131$ & $1.080+i 0.014$ \\
& & 800 & $5.570+i 0.242$ & $1.361+i 0.059$ & $0.969+i 0.173$ & $1.234+i 0.220$ \\
& & 1000 & $6.862+i 0.590$ & $1.676+i 0.144$ & $0.952+i 0.214$ & $1.468+i 0.329$ \\
\hline \multirow{3}{*}{2000} & \multirow{3}{*}{438} & 500 & $5.470+i 0.064$ & $1.081+i 0.013$ & $0.961+i 0.193$ & $1.013+i 0.203$ \\
& & 800 & $6.169+i 0.234$ & $1.219+i 0.046$ & $0.942+i 0.233$ & $1.041+i 0.258$ \\
& & 1000 & $6.941+i 0.474$ & $1.372+i 0.094$ & $0.926+i 0.263$ & $1.117+i 0.317$ \\
\hline
\end{tabular}

Table 1. Values of GS and BW parametrisations and the resulting Omnès functions at different c.m. energies for various values of $M_{\rho}\left(\Gamma_{\rho}\right)$.

\begin{tabular}{|c|c|c|c|c|}
\hline \multirow[b]{2}{*}{$\lambda_{W}-$} & \multirow[b]{2}{*}{$\lambda_{W^{+}}$} & \multicolumn{3}{|c|}{$\sigma(\mathrm{pb})$} \\
\hline & & $\begin{array}{l}P_{e^{-}}=0 \\
P_{e^{+}}=0\end{array}$ & $\begin{array}{l}P_{e^{-}}=-0.8 \\
P_{e^{+}}=+0.6\end{array}$ & $\begin{array}{c}P_{e^{-}}=0.8 \\
P_{e^{+}}=-0.6\end{array}$ \\
\hline-1 & -1 & 0.0003 & 0.0010 & 0.0 \\
\hline-1 & 0 & 0.0191 & 0.0541 & 0.0024 \\
\hline-1 & 1 & 3.4943 & 10.063 & 0.2795 \\
\hline 0 & -1 & 0.0032 & 0.0084 & 0.0011 \\
\hline 0 & 0 & 0.0468 & 0.1124 & 0.0263 \\
\hline 0 & 1 & 0.0191 & 0.0541 & 0.0024 \\
\hline 1 & -1 & 0.0921 & 0.2653 & 0.0074 \\
\hline 1 & 0 & 0.0032 & 0.0085 & 0.0011 \\
\hline 1 & 1 & 0.0003 & 0.0010 & 0.0 \\
\hline
\end{tabular}

Table 2. SM cross sections in pb for $\sqrt{s}=800 \mathrm{GeV}$ with different beam polarizations and for different $W^{+} W^{-}$helicities.

for positrons at the interaction point is proposed, with a possible upgrading to about $60 \%$ for the positron beam. As a recapitalation we will describe how the process under consideration is affected by beam polarization. The $W^{+} W^{-}$production at ILC considered here is sensitive to beam polarization, and in the following we will explain how to exploit this to our advantage. For the purpose of clarity and to set up the stage for our discussions in what will follow, we present the cross sections of $e^{+} e^{-} \rightarrow W^{+} W^{-}$at $\sqrt{s}=800 \mathrm{GeV}$ with different beam polarizations and final state polarizations, in table 2 . It can be clearly seen from the table, that the different $W$ helicities production cross section depend on initial beam polarizations. Note that the dominant $t$-channel is absent in the case of rightpolarized electron beams. 


\subsection{Longitudinal polarization}

In the case of longitudinal beam polarization, the dependence of the cross section to the polarization is usually parametrized through the degree of polarization, which is defined as $P_{l}=\left(N_{R}-N_{L}\right) /\left(N_{R}+N_{L}\right)$, where $N_{L, R}$ denote the number of left-polarized and right-polarized electrons (or positrons) respectively. For an electron beam with degree of longitudinal polarization $P_{l}$ and a positron beam with degree of polarization $P_{\bar{l}}$, the total cross section in the centre of mass frame with c.m. energy $\sqrt{s}$ is given by,

$$
\frac{d \sigma}{d \cos \theta}=\frac{\beta}{128 \pi s}\left[\left(1+P_{l}\right)\left(1-P_{\bar{l}}\right)\left|M_{+-}\right|^{2}+\left(1-P_{l}\right)\left(1+P_{\bar{l}}\right)\left|M_{-+}\right|^{2}\right]
$$

where $\beta=\sqrt{1-4 M_{W}^{2} / s}$. $\quad M_{+-}=M\left(e_{L}^{+} e_{R}^{-} \rightarrow W^{+} W^{-}\right)$is the helicity amplitude with right-handed electron and left-handed positron, and $M_{-+}=M\left(e_{R}^{+} e_{L}^{-} \rightarrow W^{+} W^{-}\right)$is the helicity amplitude with left-handed electron and right-handed positron. The helicity amplitudes we used to compute $M_{ \pm \mp}$ are those given in ref. [104] in the SM case, and the effect of SFI is introduced through the Omnès functions described in section 2.

\subsection{Transverse polarization}

At the ILC, with the help of the proposed spin rotator scheme the longitudinal beam polarization can be reoriented to achieve TP of the same degree [98]. As explained below, the transverse polarization directions can be used to define the azimuthal direction of the $W^{-}$boson produced. For arbitrary polarization of the initial beams, the expression for the differential cross section in eq. (3.1) is modified to [99, 100, 102, 105]

$$
\begin{aligned}
\frac{d \sigma}{d \Omega}=\frac{\beta}{64 \pi^{2} s} & \left\{\frac{1}{4}\left(\left(1+P_{l}\right)\left(1-P_{\bar{l}}\right)\left|M_{+-}\right|^{2}+\left(1-P_{l}\right)\left(1+P_{\bar{l}}\right)\left|M_{-+}\right|^{2}\right)\right. \\
& \left.-\frac{1}{2} P_{t} P_{\bar{t}}\left(\cos 2 \phi \operatorname{Re} M_{+-}^{*} M_{-+}-\sin 2 \phi \operatorname{Im} M_{+-}^{*} M_{-+}\right)\right\},
\end{aligned}
$$

where $\phi$ is the azimuthal angle of the $W^{-}$, in the reference frame with $x$ axis defined along the transverse polarization direction of the electron and the positron (with $P_{t, \bar{t}} \geq 0$ ). The polarization vectors of the electron and positron beams can then be written as,

$$
\vec{P}_{e^{-}}=\left(P_{t}, 0, P_{l}\right) \quad \text { and } \quad \vec{P}_{e^{+}}=\left(P_{\bar{t}}, 0, P_{\bar{l}}\right),
$$

respectively. The degrees of polarization satisfy the relation, $\left(\left|P_{t, \bar{t}}\right|^{2}+\left|P_{l, \bar{l}}\right|^{2}\right) \leq 1$. For simplicity and clarity of discussion, we consider pure transverse polarization, setting $P_{l, \bar{l}}=0$ for our analyses with TP.

\section{Numerical analyses with $W^{\prime} s$}

In this section we present our numerical analysis for the process $e^{+} e^{-} \rightarrow W^{+} W^{-}$in the presence of SFI and the other models considered in the Introduction along with the SM without SFI. We first investigate the total cross section, followed by the angular distribution of the $W^{\prime} s$, the fraction of the $W^{\prime} s$ emitted in the backward hemisphere along with the left-right asymmetry. The analyses with TP in the initial state is also considered in this section. 


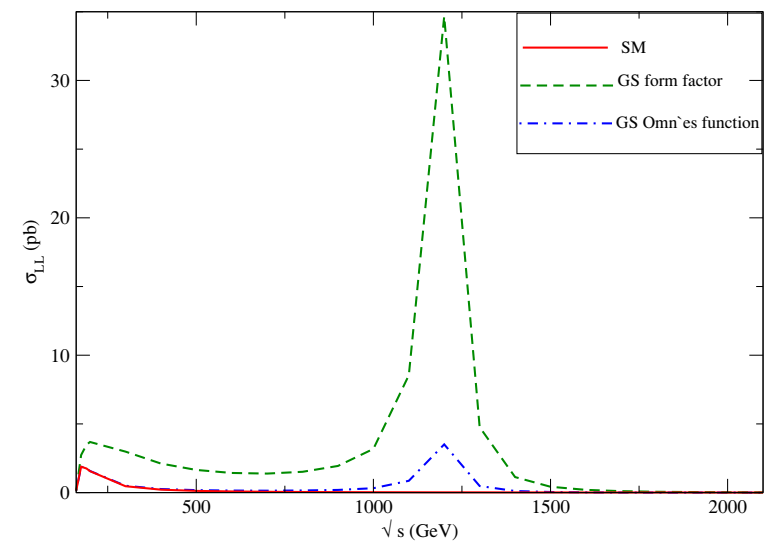

Figure 2. Total unpolarized cross section for $W_{L}$ as a function of $\sqrt{s}$ for SM, along with GS form factor and the respective Omnès function for $M_{\rho}=1200 \mathrm{GeV}, \Gamma_{\rho}=94 \mathrm{GeV}$.

\subsection{Total cross section}

We start our analyses with the first observable, where the total cross section with longitudinal beam polarization is considered, as given in eq. (3.1).

As demonstrated in section 2 the naive form factor like the GS or BW need to be improved through the description of an Omnès function. While we have demonstrated this by a comparison table in table 1 we will further consider the effect specific to $e^{+} e^{-} \rightarrow$ $W^{+} W^{-}$. Figure 2 shows the total unpolarized cross section as a function of $\sqrt{s}$ for $W_{L} W_{L}$, for a given resonance in the case of GS form factor and the relevant Omnès function obtained from it compared with the SM. We heve used $M_{\rho}=1200 \mathrm{GeV}$ and $\Gamma_{\rho}=94 \mathrm{GeV}$. In the case of the form factor itself, the large deviation present throughout the range of centre of mass energy considered, which is likely to be in conflict with the existing experimental observations (for the parameters considered here), further emphasizes the need for the present approach. Accordingly, we consider the Omnès function for our further analyses. The width, although motivated by the chiral QCD, is put in an ad hoc manner. Sensitivity of our results to the width of the resonance need to be checked. Figure 3 shows the dependence of the cross section of $W_{L} W_{L}$ production at $\sqrt{s}=800 \mathrm{GeV}$ on the width of a resonance at $M_{\rho}=1200 \mathrm{GeV}$. The cross section changes by about $1 \%$ either way from the value obtained using the width obtained from eq. (2.8), $\Gamma_{\rho}=94 \mathrm{GeV}$, demonstrating the robustness of the parametrization.

In order to understand the behaviour of the cross section with the use of different form factors as sources for $\delta$, we present in figure 4 the total cross section for beam polarizations ${ }^{2}$ of $P_{e^{-}}=0.8$ and $P_{e^{+}}=-0.6$ plotted against the c.m. energy, for three different sets of resonance parameters along with the SM expectation. When the effect of BW resonance is compared with a more complex GS form factor, the difference is not noticeable. Henceforth, we will present our results for the Omnès function obtained using a GS form factor, with judiciously (but arbitrarily) chosen $M_{\rho}=1600 \mathrm{GeV}$ and width computed from eq. (2.8).

\footnotetext{
${ }^{2}$ Throughout this article, when we consider longitudinal polarization we assume transverse polarization is absent, and therefore $\left|\vec{P}_{e^{-}}\right|=P_{e^{-}}=P_{l}$ and $\left|\vec{P}_{e^{+}}\right|=P_{e^{+}}=P_{l}$.
} 


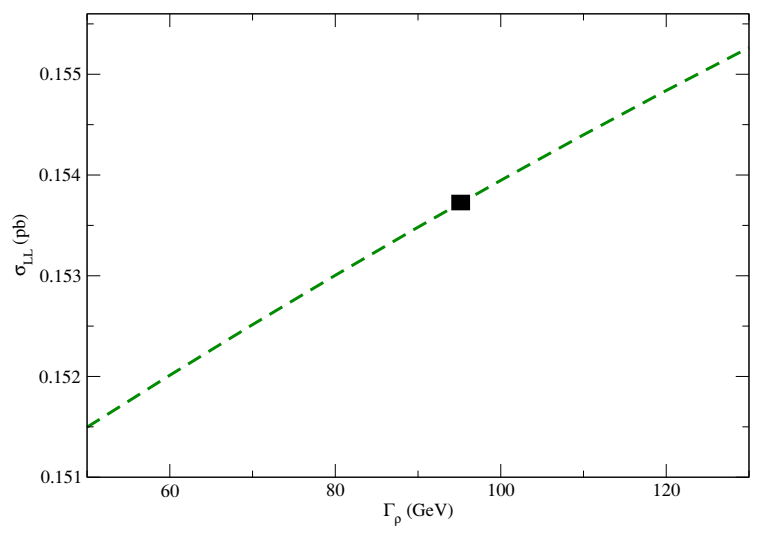

Figure 3. Total unpolarized cross section for $W_{L}$ as a function of width for $M_{\rho}=1200 \mathrm{GeV}$, at $\sqrt{s}=800 \mathrm{GeV}$. The square denotes the value of cross section for $\Gamma_{\rho}$ from eq. (2.8).

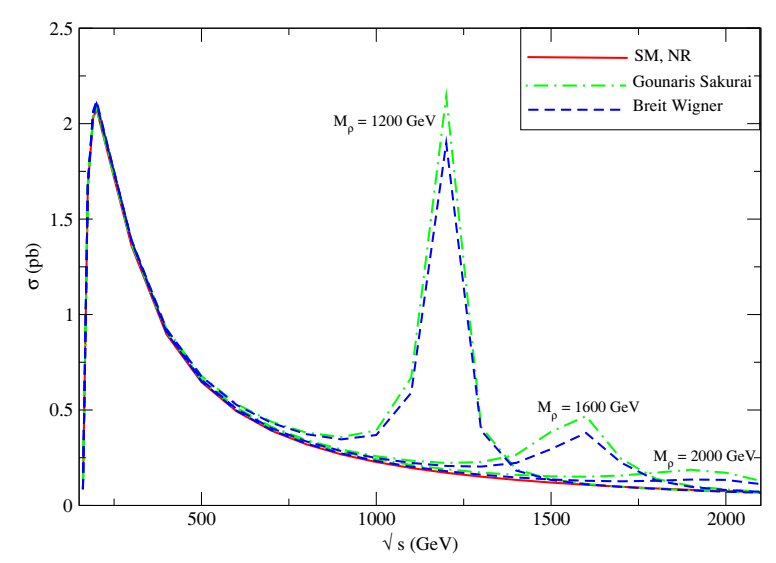

Figure 4. Total polarized cross section of unpolarized $W W$ production with $P_{e^{-}}=0.8, P_{e^{+}}=$ -0.6 , as a function of $\sqrt{s}$ for SM, NR along with GS-Omnès and BW-Omnès parametrization for different resonances.

Anticipating a symmetric, centrally peaked, $\cos \theta$ distribution of $W_{L} W_{L}$, a cut applied on the production angle $\theta$ can select the most favourable phase space. In figure 5 the total cross section of the $W_{L} W_{L}$ is presented for same beam polarizations as above, plotted against the c.m. energy with a cut of $|\cos \theta|<0.5$, with GS Omnès and Non Resonant models (NR). We have checked that compared to a situation with unpolarized beams, the contrast to the SM case is enhanced, and the sensitivity to effect of SFI is improved considerably, and likely to be observed, achievable at $\sqrt{s}=500-1000 \mathrm{GeV}$. We present the values of cross section corresponding to the case above, at $\sqrt{s}=500,800$ and $1000 \mathrm{GeV}$ in table 3. For comparison we also present the corresponding values of cross section in the case of selected $Z^{\prime}$ models discussed in Introduction.

Cross section with transversely polarized $W$ 's $\left(W_{T}\right)$ in the final state are not affected by the SFI, as is clear from our analysis in section 2 . However for the $Z^{\prime}$ models, this channel is also affected by new physics. While the effect of this in the total cross section is negligibly small, in the following we will describe some observables with $W_{T}$ in the final state. The inclusion of the initial beam polarization with $P_{e^{-}}=-0.8$ and $P_{e^{-}}=0.6$ enhances the statis- 


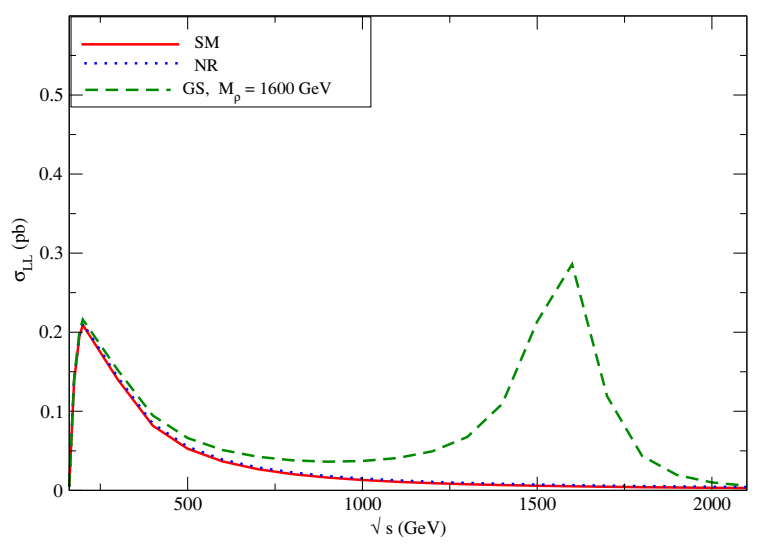

Figure 5. Cross section for $W_{L} W_{L}$, with $P_{e^{-}}=0.8, P_{e^{+}}=-0.6$, as a function of $\sqrt{s}$ in SM, NR along with GS-Omnès parametrization. An angular cut of $|\cos \theta|<0.5$ is applied.

\begin{tabular}{|c|c|c|c|c|c|}
\hline \multirow{3}{*}{$P_{e^{-}}$} & & & \multicolumn{3}{|c|}{$\sigma(\mathrm{pb})$} \\
\hline \multirow{4}{*}{0.8} & $P_{e^{+}}$ & Model & $500 \mathrm{GeV}$ & $800 \mathrm{GeV}$ & $1000 \mathrm{GeV}$ \\
\cline { 3 - 5 } & & SM & 0.053 & 0.021 & 0.013 \\
\cline { 3 - 5 } & & Omnès fn & 0.066 & 0.038 & 0.037 \\
\cline { 3 - 5 } & NR & 0.055 & 0.023 & 0.015 \\
\cline { 3 - 6 } & ALRSM & 0.044 & 0.011 & 0.002 \\
\cline { 3 - 6 } & & LRSM & 0.045 & 0.012 & 0.005 \\
\cline { 3 - 6 } & $E_{6}(\chi)$ & 0.052 & 0.020 & 0.012 \\
\cline { 3 - 6 } & LHM & 0.095 & 0.073 & 0.073 \\
\hline
\end{tabular}

Table 3. A comparison of the cross section of $W_{L} W_{L}$ production with beam polarizations of $P_{e^{-}}=0.8$ and $P_{e^{+}}=-0.6$ in the case of SFI, and with selected $Z^{\prime}$ models. A production angle cut of $|\cos \theta|<0.5$ is applied.

tics, but the new physics effects are similar to the unpolarized beam case. Henceforth we have therefore considered only $P_{e^{-}}=0.8$ and $P_{e^{-}}=-0.6$ and the case of unpolarized beams.

\subsection{Angular distribution of $W_{L} W_{L}$ and $W_{L} W_{T}$}

We now consider the angular distribution of the $W_{L}$, with both $W$ 's in the final state being longitudinally polarized in figure 6 at c.m. energy of $800 \mathrm{GeV}$, for the different scenarios considered. We have considered $M_{\rho}=1600 \mathrm{GeV}$ as our representative point here, and the initial beams are polarized. In the same figure we also plot the effect of generic $Z^{\prime}$ models considered in ref. [95], for the parameter values used in accordance with electroweak precision results. In our study for the $Z^{\prime}$ models, we consider the mixing angle $\theta_{M}=0.003$ and $\Delta M=0.12 \mathrm{GeV}$. For LHM, $f=1 \mathrm{TeV}$ and $\cos \theta_{H}=0.45$ is considered satisfying the electroweak constraints. Clearly, it is hard to distinguish between all $Z^{\prime}$ and the SFI with the angular distribution. While all the models show similar angular dependence, the $E_{6}$ and LR models demonstrate a qualitatively different behaviour compared to the SFI and LHM models, in the sense that, while the former models show a diminishing effect, 


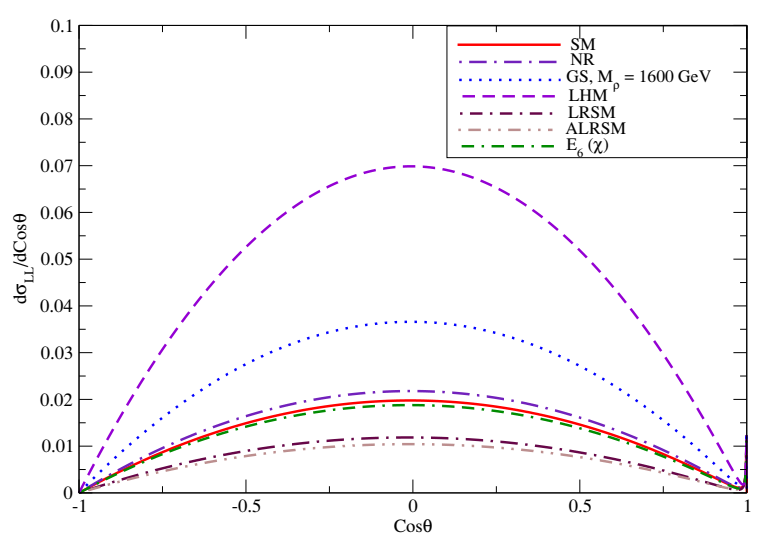

Figure 6. Polar angle distribution of $W_{L}$ in $W_{L} W_{L}$ production in SM, NR, GS-Omnès parametrization and the different $Z^{\prime}$ models, with $P_{e^{-}}=0.8$ and $P_{e^{+}}=-0.6$ at $\sqrt{s}=800 \mathrm{GeV}$.

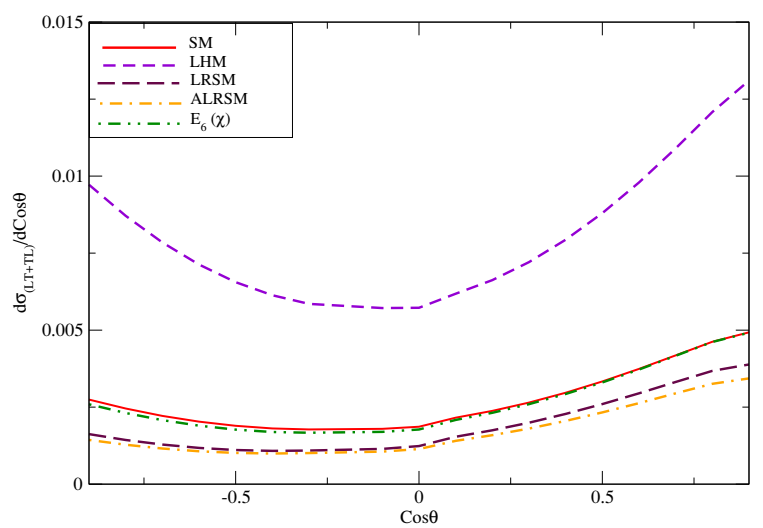

Figure 7. Polar angle distribution of $W_{L}$ in $W_{L} W_{T}+W_{T} W_{L}$ production in SM and the different $Z^{\prime}$ models with initial beam polarization of $P_{e^{-}}=0.8$ and $P_{e^{+}}=-0.6$ at $\sqrt{s}=800 \mathrm{GeV}$.

the latter models provide an enhancement in the angular distribution with respect to the SM value. We emphasize that the parameter set used in the case of $Z^{\prime}$ models were the most optimistic scenarios (with the largest possible deviation consistent with the existing experimental constraints). Therefore, it is possible to expect larger deviations than those allowed by typical $Z^{\prime}$ models here within the LHM as well as SFI. While this itself could act as a model discriminator, we notice that, the SFI is present only in the case of $W_{L} W_{L}$ final state, whereas the $Z^{\prime}$ models including the LHM can affect the case with $W_{L} W_{T}$ in the final state. When both $W^{\prime} s$ are transversely poalrized in the final state, no effect of SFI or $Z^{\prime}$ is observed. This is because $W_{T} W_{T}$, are mostly produced through the $\nu$ exchanged $t$-channel, whereas the $Z^{\prime}$ affects the $s$-channel only. Thus, with one of the $W$ transversely polarized and the other longitudinally polarized, $Z^{\prime}$ models sensitivity can be observed with no influence from SFI. Note that the $Z^{\prime}$ models including the LHM affect the process through changed SM couplings, as well as through the presence of a vector boson resonance. But the above statements are nevertheless true numerically, and has been checked explicitly. In figure 7 we plot the angular distribution of $W_{L}$ with $W_{L} W_{T}$ 


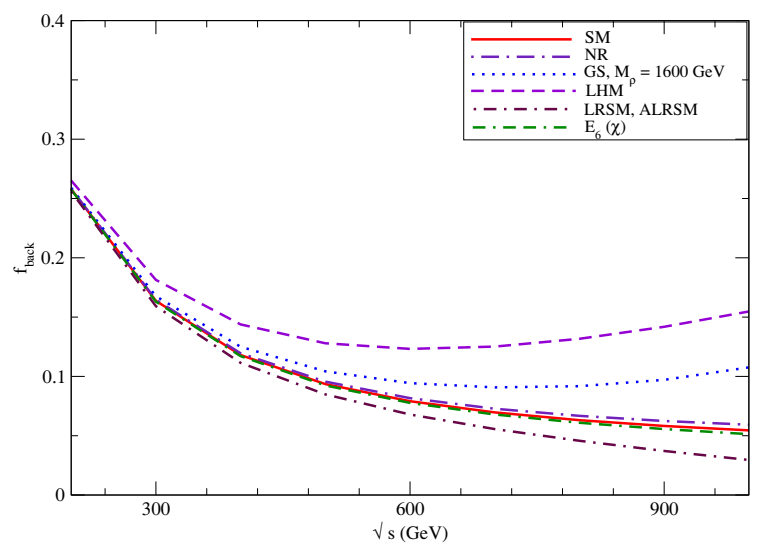

Figure 8. Fraction of unpolarized $W^{\prime} s$ emitted in the backward hemisphere as a function of $\sqrt{s}$, for the different models considered, with $P_{e^{-}}=0.8$ and $P_{e^{+}}=-0.6$.

in the final state, at the same c.m. energy of $\sqrt{s}=800 \mathrm{GeV}$ and initial polarization as in the earlier case of $W_{L} W_{L}$. Except the LHM, other models show insignificant deviation. Furthermore, the deviations are qualitatively different. Thus, results presented in figure 6 and figure 7 together will be able to distinguish between SFI, LHM and other $Z^{\prime}$ models.

\subsection{Forward backward asymmetry}

Establishing a vector resonance, and further discriminating different prospective models through the above angular distribution could be a challenge to the experiments, especially considering the small cross sections involved, and efficiencies of reconstructing final state polarizations. We therefore look next at the integrated observables. Integrating the polar angular distributions, we define the Forward-Backward (FB) asymmetry,

$$
A_{\mathrm{FB}}=\frac{\int_{-1}^{0}(d \sigma / d \cos \theta) d \cos \theta-\int_{0}^{1}(d \sigma / d \cos \theta) d \cos \theta}{\int_{-1}^{1}(d \sigma / d \cos \theta) d \cos \theta}
$$

or, equivalently, the fraction of the $W$ 's emitted in the backward hemisphere,

$$
f_{\text {back }}=\frac{\int_{-1}^{0}(d \sigma / d \cos \theta) d \cos \theta}{\int_{-1}^{1}(d \sigma / d \cos \theta) d \cos \theta}
$$

These two observables are related to each other by $A_{\mathrm{FB}}=2 f_{\text {back }}-1$. Figure 8 presents $f_{\text {back }}$ of the unpolarized $W^{\prime} s$ with initial beam polarization of $P_{e^{-}}=0.8$ and $P_{e^{+}}=-0.6$. The behaviour is similar to the case of angular distributions, with a diminishing effect from $Z^{\prime}$ models other than LHM, while the SFI and LHM showing an enhancement. Reading from table 4 , at $\sqrt{s}=800 \mathrm{GeV}, 6 \%$ of the events are in the backward region. In the presence of SFI, this is substantially increased to a $9 \%$, while for LHM it is more than doubled to $13 \%$. Other $Z^{\prime}$ models have smaller effect with LRSM and ALRSM showing about $4-5 \%$, while $E_{6}(\chi)$ remaining at $6 \%$. Recall that we have not considered any angular cut here. As in the case of total cross section discussed above, an angular cut will considerably enhance this effect, with comparatively smaller cost in terms of statistics. Also it may be noted 


\begin{tabular}{|c|c|c|c|c|c|c|c|c|c|}
\hline \multirow[b]{2}{*}{$P_{e^{-}}$} & \multirow[b]{2}{*}{$P_{e^{+}}$} & \multirow[b]{2}{*}{ Model } & \multirow[b]{2}{*}{$\begin{array}{c}M_{\rho} \\
(\mathrm{GeV})\end{array}$} & \multicolumn{3}{|c|}{$f_{\text {back }}\left(W_{\text {unp }}\right)$} & \multicolumn{3}{|c|}{$f_{\text {back }}\left(W_{L T+T L}\right)$} \\
\hline & & & & $\begin{array}{c}500 \\
(\mathrm{GeV})\end{array}$ & $\begin{array}{c}800 \\
(\mathrm{GeV})\end{array}$ & $\begin{array}{c}1000 \\
(\mathrm{GeV})\end{array}$ & $\begin{array}{c}500 \\
(\mathrm{GeV})\end{array}$ & $\begin{array}{c}800 \\
(\mathrm{GeV})\end{array}$ & $\begin{array}{c}1000 \\
(\mathrm{GeV})\end{array}$ \\
\hline \multirow{7}{*}{0} & \multirow{7}{*}{0} & $\mathrm{SM}$ & & 0.037 & 0.024 & 0.021 & 0.110 & 0.099 & 0.097 \\
\hline & & Omnès fn. & 1600 & 0.039 & 0.030 & 0.031 & 0.110 & 0.099 & 0.097 \\
\hline & & NR & & 0.037 & 0.025 & 0.022 & 0.110 & 0.099 & 0.097 \\
\hline & & ALRSM & 1600 & 0.035 & 0.022 & 0.017 & 0.102 & 0.077 & 0.059 \\
\hline & & LRSM & 1600 & 0.037 & 0.025 & 0.022 & 0.111 & 0.102 & 0.104 \\
\hline & & $E_{6}(\chi)$ & 1600 & 0.037 & 0.027 & 0.025 & 0.114 & 0.113 & 0.123 \\
\hline & & LHM & 1550 & 0.036 & 0.024 & 0.021 & 0.108 & 0.104 & 0.117 \\
\hline \multirow{7}{*}{0.8} & \multirow{7}{*}{-0.6} & SM & & 0.094 & 0.063 & 0.055 & 0.306 & 0.298 & 0.295 \\
\hline & & Omnès fn. & 1600 & 0.104 & 0.092 & 0.108 & 0.306 & 0.298 & 0.295 \\
\hline & & NR & & 0.096 & 0.067 & 0.059 & 0.306 & 0.298 & 0.0295 \\
\hline & & ALRSM & 1600 & 0.083 & 0.042 & 0.024 & 0.142 & 0.286 & 0.228 \\
\hline & & LRSM & 1600 & 0.085 & 0.046 & 0.030 & 0.287 & 0.234 & 0.167 \\
\hline & & $E_{6}(\chi)$ & 1600 & 0.092 & 0.061 & 0.051 & 0.303 & 0.288 & 0.276 \\
\hline & & LHM & 1550 & 0.128 & 0.132 & 0.155 & 0.361 & 0.410 & 0.434 \\
\hline
\end{tabular}

Table 4. Fraction of unpolarized $W$ 's and $W_{L} W_{T}+W_{T} W_{L}$ emitted in the backward direction for different polarization combinations at different c.m. energies.

that, the small fractions shown here are a little deceptive. With the cross section at $0.3 \mathrm{pb}$, for a moderate integrated luminosity of $100 \mathrm{fb}^{-1}, 6 \%$ of the events amount to about 2000 events. Even after putting in a BR $(\sim 4 / 27$ for semi-leptonic channel) and reconstruction efficiency $(\sim 65 \%)$, a few hundred events will remain. Certainly, a measurement of increase by $9 \%$ or $13 \%$ is conceivable in this case. At $\sqrt{s}=500 \mathrm{GeV}$, the deviations are smaller. SFI improves the fraction to $10.4 \%$ from the SM value of $9.4 \%$, while LHM deviates to a much larger $12.8 \%$. Other models reduces the fraction with ALRSM giving the largest fraction of 8.3\%. Again, the cross section is increased by a few times, but, it is perhaps very difficult to see the effect at a per-cent level. It is possible to see the effect of LHM at high luminosity. Without beam polarization, as presented in table 4 , the SFI improves the $2.4 \%$ value of SM to a $3 \%$, while all other models show smaller effects. With a total cross section about $3.7 \mathrm{pb}$, the statistics is improved by an order of magnitude. Even then this will require a somewhat larger luminosity to make any meaningful analysis. At $\sqrt{s}=500 \mathrm{GeV}$, there is no observable deviation for any models. In the case of $\sqrt{s}=1000 \mathrm{GeV}$, the picture is very similar to that of $\sqrt{s}=800 \mathrm{GeV}$, with some marginal improvement for all models except ALRSM. In the case of ALRSM, the effect is comparable to that of SFI, but while in the former case it is a diminishing effect, the latter case is an enhancement. The advantage of beam polarization is evident from the above analysis.

In table 4 we also present the case of $W_{L} W_{T}$. As expected there is no effect of SFI here, as it affects only $W_{L} W_{L}$ channel. With unpolarized beams, except the ALRSM, which 


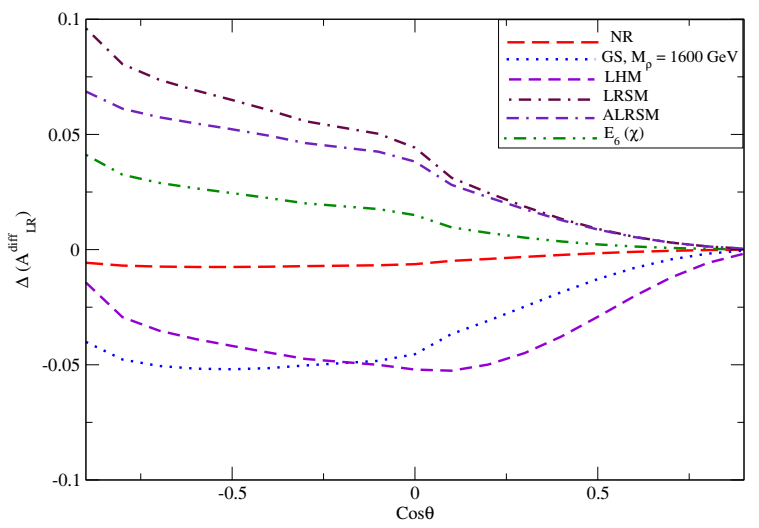

Figure 9. Deviations in the differential LR asymmetry for GS, NR and the various $Z^{\prime}$ models from $\mathrm{SM}$ as a function of $\cos \theta$, at $\sqrt{s}=800 \mathrm{GeV}$.

shows a change of $2 \%$ in the fraction at $\sqrt{s}=800 \mathrm{GeV}$ and about $4 \%$ at $\sqrt{s}=1000 \mathrm{GeV}$, all models show very small effects. Even these effects of ALRSM are not very promising. Thus, practically, there is no effect from any models, and thus, a comparison of unpolarized $W$ with $W_{L} W_{T}$ is not very illuminating from the point of model discrimination. The situation is changed for better with the beam polarization considered. Largest deviation is in the LHM taking the SM value of the fraction of about $29.8 \%$ to $41 \%$ at $\sqrt{s}=800 \mathrm{GeV}$ and to $43.4 \%$ at $\sqrt{s}=1000 \mathrm{GeV}$. Even such large deviations may not be visible owing to very small cross section available in this channel. Compared to the unpolarized $W$ 's, the cross section is reduced by a factor of about 50 , and we need to also consider the efficiency of $W$ polarization measurement. So, overall the number of events at $100 \mathrm{fb}^{-1}$ may even be smaller than 10. This will certainly need very high luminosity to make any statement. The efficiency to measure polarization of both $W^{\prime} s$ from an event is small, compared to the case with polarization of only one of it measured. It will be beneficial if one considers the case, where one of the $W$ is longitudinally polarized, and the other unpolarized. In $W_{L} W_{L+T}$, only about $12 \%$ of the contribution comes from $W_{L} W_{T}$, as seen from table 2 , in case of $P_{e^{-}}=0.8$ and $P_{e^{+}}=-0.6$. The behavioural pattern will therefore be the same as $W_{L} W_{L}$, due to which we do not present the results here.

\subsection{Left right (LR) asymmetry}

We now turn our attention to an asymmetry constructed with the help of beam polarization. The differential left-right asymmetry is defined as

$$
A_{L R}^{\mathrm{diff}}=\frac{d \sigma\left(e_{R}^{+} e_{L}^{-}\right) / d \cos \theta-d \sigma\left(e_{L}^{+} e_{R}^{-}\right) / d \cos \theta}{d \sigma\left(e_{R}^{+} e_{L}^{-}\right) / d \cos \theta+d \sigma\left(e_{L}^{+} e_{R}^{-}\right) / d \cos \theta}
$$

where $\theta$ is the $W$ scattering angle. In figure 9 , we plot the deviation from the SM case,

$$
\Delta A_{L R}^{\text {diff }}=\frac{A_{L R}^{\text {diff }}(\text { new })-A_{L R}^{\text {diff }}(\mathrm{SM})}{A_{L R}^{\text {diff }}(\mathrm{SM})}
$$

as a function of $\cos \theta$ for the different models considered, at $\sqrt{s}=800 \mathrm{GeV}$. Clearly, the qualitatively different features are potential model discriminators. In a role reversal com- 


\begin{tabular}{|c|c|c|c|c|c|c|}
\hline \multirow{2}{*}{ Model } & \multicolumn{3}{|c|}{$A_{L R}\left(W_{\text {up }} W_{\text {up }}\right)$} & \multicolumn{3}{c|}{$A_{L R}\left(W_{L} W_{\text {up }}\right)$} \\
\cline { 2 - 7 } & $\begin{array}{c}500 \\
(\mathrm{GeV})\end{array}$ & $\begin{array}{c}800 \\
(\mathrm{GeV})\end{array}$ & $\begin{array}{c}1000 \\
(\mathrm{GeV})\end{array}$ & $\begin{array}{c}500 \\
(\mathrm{GeV})\end{array}$ & $\begin{array}{c}800 \\
(\mathrm{GeV})\end{array}$ & $\begin{array}{c}1000 \\
(\mathrm{GeV})\end{array}$ \\
\hline SM & 0.891 & 0.898 & 0.900 & 0.705 & 0.678 & 0.671 \\
\hline Omnès fn. & 0.877 & 0.853 & 0.819 & 0.697 & 0.669 & 0.662 \\
\hline NR & 0.887 & 0.890 & 0.890 & 0.702 & 0.676 & 0.669 \\
\hline ALRSM & 0.938 & 0.968 & 0.988 & 0.738 & 0.744 & 0.814 \\
\hline LRSM & 0.911 & 0.950 & 0.982 & 0.763 & 0.850 & 0.948 \\
\hline$E_{6}(\chi)$ & 0.898 & 0.916 & 0.933 & 0.739 & 0.777 & 0.838 \\
\hline LHM & 0.866 & 0.851 & 0.839 & 0.766 & 0.793 & 0.800 \\
\hline
\end{tabular}

Table 5. The integrated left-right polarization asymmetry in the backward hemisphere with both $W$ 's unpolarized, and with one of the $W$ longitudinally polarized, while the other is unpolarized, for different c.m. energies.

pared to the earlier observables, here the $Z^{\prime}$ models show enhancement in the asymmetry compared to the SM case, while the LHM and the SFI show a diminishing effect. The above analyses was done with idealistic beam polarization. More realistic computations with $\left|P_{e^{-}}\right|=\left|P_{e^{+}}\right| \neq 1$ can be done in the future.

However it is worth noting, within the SM, at $500 \mathrm{fb}^{-1}$ lumunosity about 12500 events are expected in the backward hemisphere for $P_{e^{-}}=-0.8$ and $P_{e^{+}}=0.6$, and about 1000 events in the case of $P_{e^{-}}=0.8$ and $P_{e^{+}}=-0.6$ in the semileptonic channel with a detection efficiency of about $65 \%$. This leads to an asymmetric number of events of about a 11500 , and a $5 \%$ change in this, as is the case with SFI, is very likely to be measurable. If we can measure the asymmetry at 1-2\% level, it is possible to distinguish between the SFI and LHM through a comparison of asymmetries in the forward and backward hemispheres, or even just by considering only the backward region. The differential asymmetry (eq. (4.3)) integrated over the forward hemisphere at three different c.m. energies are presented in table 5. The effect of SFI is doubled to about $10 \%$ deviation from the SM case at $\sqrt{s}=$ $1000 \mathrm{GeV}$ with similar increase in other models, where discrimination of models is even more promising, although the actual number of events will come down.

\subsection{Azimuthal distribution of $W^{\prime} s$}

We now construct some observables with TP, discussed in section 3.2. Notice that $M_{ \pm \mp}$ in eq. (3.2) are computed by setting $\phi=0$, and thus automatically reproduce the null theorem, stating that the cross section with transversely polarized beams averaged over the azimuthal angle is the same as the cross section with unpolarized beams, in agreement with the arguments presented for general $e^{+} e^{-}$collisions with chirality conserving interactions (massless electrons) [105]. We have suppressed the helicity information of final state $W$ 's in the invariant amplitudes defined in eq. (3.2). The SFI affects the $l=1$ partial wave amplitude of $M_{ \pm \mp}^{L L}$ for the longitudinal $W$ 's. TP case differs from the unpolarized and longitudinally polarized beam case through the interference of the two amplitudes, $M_{+-} M_{-+}^{*}$. 


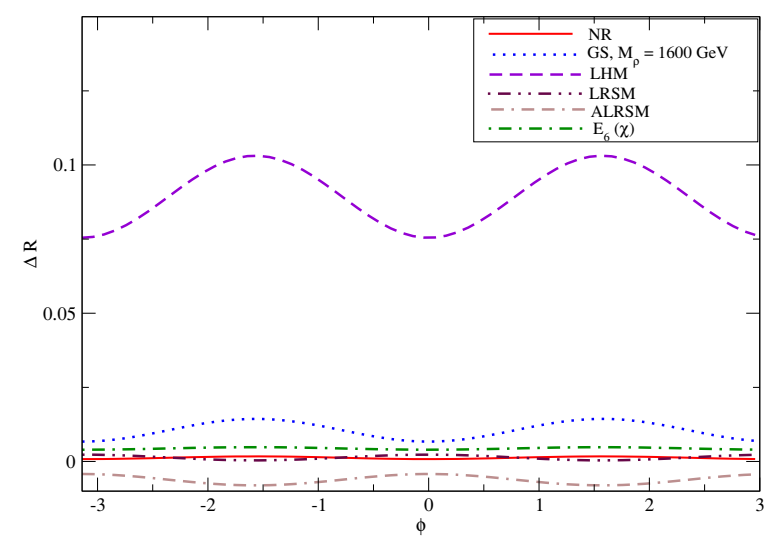

Figure 10. $\phi$ distribution of unpolarized $W^{\prime} s$ showing the deviation from SM as a function of $\phi$ at $\sqrt{s}=800 \mathrm{GeV}$, in the different scenarios considered. Purely transversely polarized beams with $P_{T}=0.8$ and $P_{\bar{T}}=0.6$ are considered.

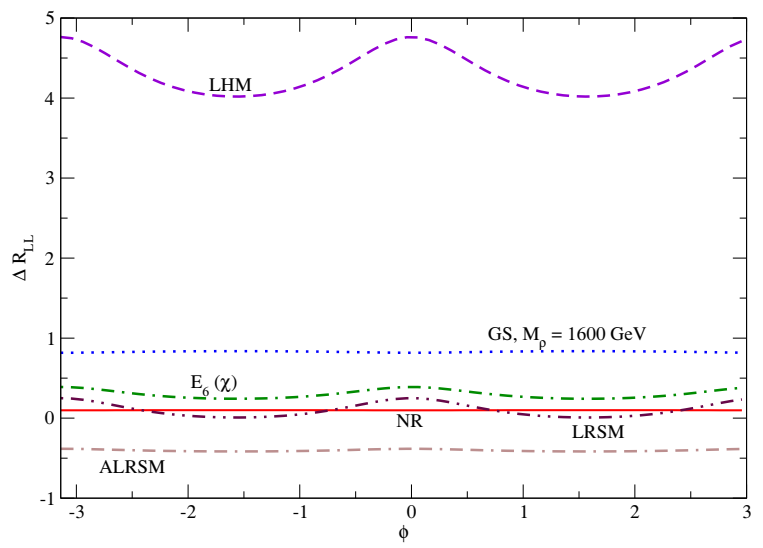

Figure 11. $\phi$ distribution of polarized $W_{L} W_{L}$ showing the deviation from SM as a function of $\phi$ at $\sqrt{s}=800 \mathrm{GeV}$, in the different scenarios considered. Purely transversely polarized beams with $P_{T}=0.8$ and $P_{\bar{T}}=0.6$ are considered.

In figure 10 we present the deviation from SM in azimuthal distribution for different scenarios considered with unpolarized $W^{\prime} s$ in the final state, where we define:

$$
\Delta R=\frac{\left.\frac{d \sigma}{d \phi}\right|_{\text {new }}}{\left.\frac{d \sigma}{d \phi}\right|_{\mathrm{SM}}}-1
$$

The NR models are indistinguishable from SM, whereas SFI shows about $2 \%$ deviation. In case of the $Z^{\prime}$ models, LHM has the most significant effect with about $7-10 \%$ deviation, but the other $Z^{\prime}$ models have least significant effect. Note that what is plotted is the deviation from the SM case. Therefore the $\cos \phi$ modulation actually shows a $\phi$ dependance different from that in the SM case. This modulation itself is about $2 \%$ in the case of LHM, while the effect is negligible for all other models. When the polarization of $W$ is considered, in the presence of TP, the new physics effects are significantly enhanced. Figure 11 shows the above deviation, eq. (4.5) for all the scenarios with $W_{L} W_{L}$ in the final state. It is the most 


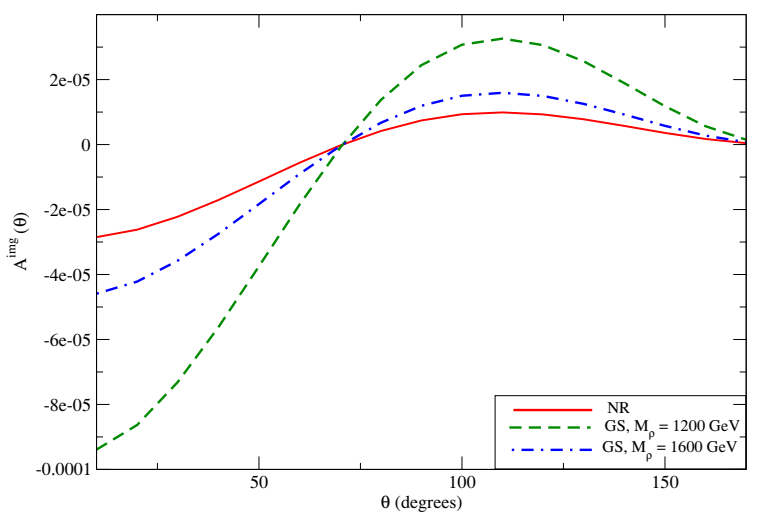

Figure 12. Asymmetry showing the contribution from imaginary part of NR, GS for different $M_{\rho}$, in presence of TP.

sensitive channel, to look for effects of new physics. However measurement through this channel depends on the efficiency of the $W$ polarization measurement as discussed before, and a very high luminosity. The alternative will be to measure the polarization of one of the $W^{\prime} s$ and select the events with $W_{L}$ in the final state. An analyses of this channel will also enhance the new physics effect.

The TP case has an interesting feature of receiving contribution from the imaginary part of the amplitude, which is only present in case of SFI. The size of the contribution from $\operatorname{Im}\left(M_{+-}^{*} M_{-+}\right)$in eq. (3.2), due to SFI can be estimated by considering the following asymmetry:

$$
A^{\mathrm{img}}(\theta)=\int_{-\pi}^{\pi} \frac{d \sigma}{d \Omega} \sin 2 \phi d \phi
$$

Figure 12, shows the contribution from the imaginary part of the amplitude, in case of unpolarized $W^{\prime} s$. It can be seen the imaginary part is too small to be measured, unless we have a very high luminosity, and we are very close to the resonance. The contribution from the imaginary part can be measured by other methods, which will be discussed in the next section.

\section{Inclusion of decays}

The observables which were considered with unpolarized and longitudinal beams, are only sensitive to the modulus of the Omnès function. The inclusion of TP addresses the problem of sensitivity to the imaginary part of the Omnès function. However we have shown that it is too small to be seen at design ILC energies. In prior work $[93,106]$, the azimuthal angles of the fermions from $W^{+} W^{-}$decay in the $W$ rest frame, has been considered in certain correlation sensitive to the effect of SFI. We now study this effect at ILC energy with LP beams. Furthermore we comment on the energy distribution also.

\subsection{Azimuthal distribution of the decay leptons}

It has been pointed out in [93] inspired by the earlier work of [106] that a correlation proportional to $\sin \left(\phi_{-}-\phi+\right)$, would be a useful indicator to pick up the signal due to 


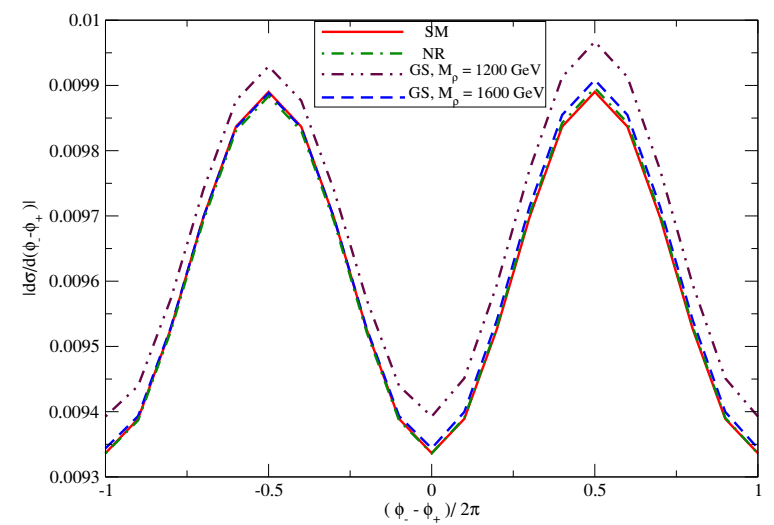

Figure 13. Expected angular distribution, with and without SFI, with initial beam polarization of $P_{e^{-}}=-0.8$ and $P_{e^{+}}=0.6$.

the imaginary part of the SFI. This is due to the fact that it is a T-odd quantity, which picks out the longitudinal-transverse spin-spin correlations, that is obtained by correlating the azimuthal angles of the $W$ decay product. The presence of non-resonant background at high energies is studied by [106] and [93] have not reported any results. Thus the question of using this quantity at the ILC remains. We have therefore carried out this study, both with unpolarized as well as longitudinally polarized beams. The results are given in figure 13, for initial beam polarization of $P_{e^{-}}=-0.8$ and $P_{e^{+}}=0.6$, for SM, NR and SFI with $M_{\rho}=1200,1600 \mathrm{GeV}$, at $\sqrt{s}=800 \mathrm{GeV}$. The effects due to SFI, can be seen from the asymmetrical nature of the peaks. It may be observed that in the absence of SFI, the curve is a $\sin \left(\phi_{-}-\phi+\right)$. In the presence of SFI, it may be observed that neighbouring peaks do not have the same height. The phase will distinctly reveal its mark, at higher c.m. energies near the resonance.

\subsection{Energy distribution of the decay leptons}

The other observable that we consider is the single energy distribution of the emitted leptons, first considered in ref. [107]. In figure 14 we present the energy distribution of the decay lepton coming from $W_{L}^{-}$, keeping the other $W$ unpolarized and allowing it to decay into anything. The qualitative features are very similar to the $\cos \theta$ distribution (figure 6), but certainly provides us with another observational tool to understand the dynamics. The $E_{6}$ models present a diminishing effect compared to the SM case, while the LHM and the SFI cases go the other way. The case discussed has used beams of polarizations $P_{e^{-}}=0.8$ and $P_{e^{-}}=-0.6$ at $\sqrt{s}=800 \mathrm{GeV}$. For the case of unpolarized beams, features remain more or less the same, but with an asymmetric distributions, as expected.

The dimensionless variable $X_{ \pm}$is defined as:

$$
X_{l^{ \pm}}=\frac{2}{\beta \sqrt{s}}\left(E_{l^{ \pm}}-\frac{\sqrt{s}}{4}(1-\beta)\right) .
$$

where $X_{l^{ \pm}}$varies between 0 and 1 , and $E_{l^{ \pm}}$is the energy of the emitted leptons. 


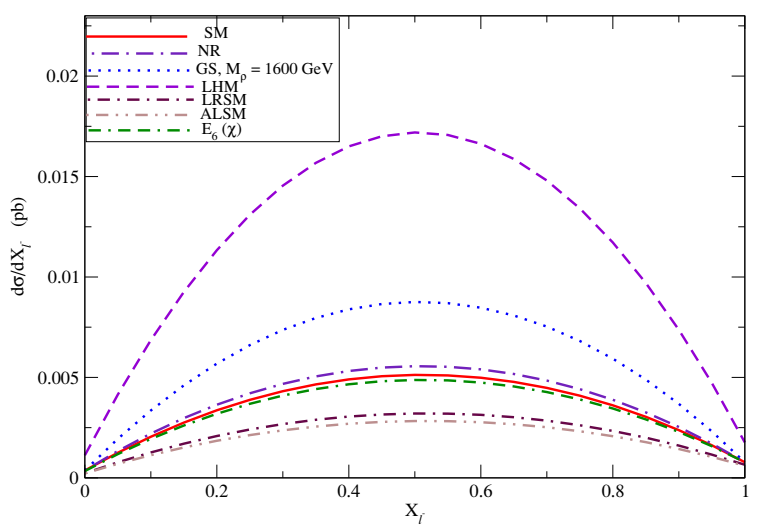

Figure 14. Laboratory energy distribution of the secondary lepton from $W_{L}^{-}$, while $W_{\text {unpol }}^{+}$is allowed to decay into anything. Longitudinally polarized beams with $P_{e^{-}}=0.8$ and $P_{e^{+}}=-0.6$ at $\sqrt{s}=800 \mathrm{GeV}$ is considered.

\section{Discussions and conclusions}

In this work we have considered the possibility of a strongly interacting $\mathrm{W}$ gauge boson sector, which is a distinct possibility in the event the Higgs mass is very high $(\geq 1 \mathrm{TeV})$ or in the event that the Higgs is light $\sim 125 \mathrm{GeV}$, consistent with the regions so far not excluded by the LHC searches. We have focussed on the fingerprinting of such a strongly interacting sector at the ILC with polarized beams, both longitudinal as well as transverse. The method we have used is the inclusion of a phase due to the SFI which modifies the $l=1$ partial wave. The phase is modelled in terms of GS or BW parametrizations due to the possibility of a resonance, or in terms of a non-resonant background. We have extended treatments available in the literature and have studied them in great detail, comparing and contrasting the results as and when it has been found necessary. The inclusion of the phase has been done following the elegant and simplified treatment of [92] which we have duly corrected here. The treatment is consistent with that in [91].

We have analyzed the process in great detail for the case of both initial as well as final state polarizations. Since the main effect resides in the dynamics of the longitudinal $W$ bosons, we have paid attention to this matter.

We have studied various observables like the total cross section, angular distribution of $W_{L}, W_{T}$, the FB as well as the LR asymmetry. The main effects are seen in the state when the polarization of $W^{\prime} s$ are studied, specially in $W_{L}$ channel, with the initial longitudinal beam polarization of $P_{e^{-}}=0.8$ and $P_{e^{+}}=-0.6$. For much of the work we have stayed with $W^{\prime} s$ in the final state. The reason for this is to obtain an analytic insight into the behaviour. In particular, for the case of transverse polarization, the formalism of Hikasa remains transparent with $W^{\prime} s$ in the final state.

In order to make our work compelling, we have carried out a detailed comparison with the popular models where new physics can arise due to the presence of an additional heavy gauge boson $Z^{\prime}$. The behavioral pattern is almost the same with $W_{L} W_{T}$ channel acting as a model discriminator for the $Z^{\prime}$ models from SFI. For almost all the observables considered 
with unpolarized and longitudinal beams, LHM behaves similar to SFI, whereas the other $Z^{\prime}$ models have the opposite effect.

We have also considered the case of decays sticking to interesting results that can be obtained using analytic methods: this is the case of the correlation that is proportional to $\left(\phi_{-}-\phi_{+}\right)$. The inspiration comes from [93] and from the work of [106]. However, we have also included the effects of longitudinal beam polarization.

Our work shows that in order to make greater progress, more knowledge is necessary on the nature of the strong interaction. Perhaps the discovery of resonances at LHC will shed light on this sector. With such information from LHC, ILC will be able to disentangle some of the contesting models against SFI. In the absence of any information from LHC, it will be a difficult job requiring very large luminosity and large c.m. energy.

Our work also shows that a strong polarization program at the ILC is very useful in shedding light on the dynamics of EWSB.

\section{A Form factor parametrizations}

In this appendix we summarize the different parametrizations that we have used in this work, given the mass and width of the resonance. We also present the expression for the non-resonance SFI formula. The knowledge of the underlying theory of strong interaction can only predict whether a resonance exists in a particular channel or not. There have been different approaches in the literature to predict resonances. One of the approach is when an effective lagrangian assuming the existence of a resonance in a particular channel is assumed and written down. Another approach N/D assumes the existence of resonance, but not with effective lagrangian models. The other way is starting from the low energy expansion of the $W W$ scattering amplitude, in analogy to pion scattering. Since in TeV region, these results violate the partial wave unitarity, there are several extrapolation schemes from low energy physics which satisfy unitarity. The method of unitarization decides whether a resonance exits in a particular channel or not.

\section{A.1 Non-resonant SFI}

$\mathrm{K}$ matrix is one of the extrapolation schemes, which in the zeroth order presents a model with a non resonant $l=I=1$ partial wave. $\mathrm{K}$ matrix unitarization in terms of partial wave amplitudes is given by:

$$
a^{(k)}(s)=\frac{a^{(0)}(s)}{1-i a^{(0)}(s)} .
$$

We have also used the form factor parametrization, where the equilvalence theorem is used that relates the scattering amplitude among longitudinal gauge bosons and the Goldstone bosons. The born cross section for the gauge boson pair production is multiplied by the form factor obtained from various models described below. 


\section{A.2 Gounaris-Sakurai parametrization}

The form factor is given in the GS parametrization as:

$$
F_{W}(S)=\frac{k\left(m_{V}^{2}\right)^{3} \sqrt{s}\left(m_{V}^{2}+d m_{V} \Gamma_{V}\right)}{k\left(m_{V}^{2}\right)^{3} \sqrt{s}\left(m_{V}^{2}-s\right)+\sqrt{s} \Gamma_{V} m_{V}^{2} g(s)-i m_{V}^{2} \Gamma_{V} k(s)^{3}},
$$

where

$$
\begin{aligned}
k(s) & =\sqrt{\frac{s}{4} \beta(s)^{2}}, \\
\beta(s) & =\sqrt{1-\frac{4 m_{W}^{2}}{s}}, \\
d & =\frac{3}{\pi} \frac{m_{W}^{2}}{k\left(m_{V}^{2}\right)^{2}} \log \left[\frac{m_{V}+2 k\left(m_{V}^{2}\right)}{2 M_{W}}\right]+\frac{m_{V}}{2 \pi k\left(m_{V}^{2}\right)}-\frac{m_{W}^{2} m_{V}}{\pi k\left(m_{V}^{2}\right)^{3}}
\end{aligned}
$$

and

$$
g(s)=k(s)^{2}\left(h(s)-h\left(m_{V}^{2}\right)\right)+k\left(m_{V}^{2}\right)^{2} h^{\prime}\left(m_{V}^{2}\right)\left(m_{V}^{2}-s\right)
$$

with

$$
h(s)=\frac{\beta(s)}{\pi} \log \left[\frac{\sqrt{s}(1+\beta(s))}{2 m_{W}}\right] .
$$

\section{A.3 Breit-Wigner parametrization}

The form factor is given in the BW parametrization as

$$
F(s)=\frac{\beta\left(m_{V}^{2}\right)^{3}\left(s-m_{V}^{2}\right)}{\beta\left(m_{V}^{2}\right)^{3}\left(s-m_{V}^{2}\right)+i \Gamma_{V} m_{V} \beta(s)^{3}},
$$

where

$$
\beta(x)=\left(1-\frac{4 m_{W}^{2}}{x}\right)
$$

\section{Acknowledgments}

We thank Prof. S.D. Rindani and Prof. L.M. Sehgal for insightful discussions at various levels. We thank G. Moortgat-Pick for useful discussions on the subject. BA thanks the Homi Bhabha Fellowships Council for partial support, and the Department of Science and Technology, Government of India for support during the course of this investigation. BA also thanks the Department of Physics, Indian Institute of Technology, Guwahati for its hospitality when part of this work was carried out. PP acknowledges the support of BRNS, DAE, Government of India (Project No.: 2010/37P/49/BRNS/1446), and the support of the DPG through the SFB (grant SFB 676/1-2006).

Open Access. This article is distributed under the terms of the Creative Commons Attribution License which permits any use, distribution and reproduction in any medium, provided the original author(s) and source are credited. 


\section{References}

[1] ATLAS collaboration, Search for a standard model Higgs boson in the $H \rightarrow Z Z \rightarrow l^{+} l^{-} \nu \bar{\nu}$ decay channel with the ATLAS detector, Phys. Rev. Lett. 107 (2011) 221802 [arXiv:1109.3357] [INSPIRE].

[2] ATLAS collaboration, Search for the standard model Higgs boson in the two photon decay channel with the ATLAS detector at the LHC, Phys. Lett. B 705 (2011) 452 [arXiv:1108.5895] [INSPIRE].

[3] ATLAS collaboration, G. Aad et al., Search for a heavy standard model Higgs boson in the channel $H \rightarrow Z Z \rightarrow l l q q$ using the ATLAS detector, Phys. Lett. B 707 (2012) 27 [arXiv:1108.5064] [INSPIRE].

[4] D. Trocino, A search for the Higgs boson in $H \rightarrow Z Z \rightarrow 2 \ell 2 \nu$ mode, arXiv:1110.1938 [INSPIRE].

[5] C. Palmer, A search for the Higgs boson in the $H \rightarrow \gamma \gamma$ channel with CMS, arXiv:1109.6805 [INSPIRE].

[6] ATLAS and CMS collaborations, Combined standard model Higgs boson searches with up to 2.3 $\mathrm{fb}^{-1}$ of pp collisions at $\sqrt{s}=7 \mathrm{TeV}$ at the LHC, ATLAS-CONF-2011-157, CMS-PAS-HIG-11-023 (2011).

[7] ATLAS collaboration, Combination of Higgs boson searches with up to $4.9 \mathrm{fb}^{-1}$ of $\mathrm{pp}$ collisions data taken at a center-of-mass energy of 7 TeV with the ATLAS experiment at the LHC, ATLAS-CONF-2011-163 (2011).

[8] CMS collaboration, Combination of CMS searches for a standard model Higgs boson, CMS-PAS-HIG-11-032 (2011).

[9] M.S. Chanowitz and M.K. Gaillard, The TeV physics of strongly interacting W's and Z's, Nucl. Phys. B 261 (1985) 379 [InSPIRE].

[10] Y.-P. Yao and C.P. Yuan, Modification of the equivalence theorem due to loop corrections, Phys. Rev. D 38 (1988) 2237 [INSPIRE].

[11] J. Bagger and C. Schmidt, Equivalence theorem redux, Phys. Rev. D 41 (1990) 264 [INSPIRE].

[12] J.M. Cornwall, D.N. Levin and G. Tiktopoulos, Uniqueness of spontaneously broken gauge theories, Phys. Rev. Lett. 30 (1973) 1268 [Erratum ibid. 31 (1973) 572] [INSPIRE].

[13] C.E. Vayonakis, Born helicity amplitudes and cross-sections in nonAbelian gauge theories, Lett. Nuovo Cim. 17 (1976) 383 [INSPIRE].

[14] T. Appelquist and C.W. Bernard, Strongly interacting Higgs bosons, Phys. Rev. D 22 (1980) 200 [InSPIRE].

[15] A.C. Longhitano, Heavy Higgs bosons in the Weinberg-Salam model, Phys. Rev. D 22 (1980) 1166 [INSPIRE].

[16] A.C. Longhitano, Low-energy impact of a heavy Higgs boson sector, Nucl. Phys. B 188 (1981) 118 [inSPIRE].

[17] T. Appelquist and G.-H. Wu, The electroweak chiral Lagrangian and new precision measurements, Phys. Rev. D 48 (1993) 3235 [hep-ph/9304240] [INSPIRE]. 
[18] ATLAS collaboration, ATLAS detector and physics performance: technical design report. 2, CERN-LHCC-99-015 (1999).

[19] CMS collaboration, CMS technical design report. Volume II: Physics performance, CERN-LHCC-2006-021 (2006) [INSPIRE].

[20] R.S. Chivukula, R. Rosenfeld, E.H. Simmons and J. Terning, Strongly coupled electroweak symmetry breaking: implication of models, hep-ph/9503202 [INSPIRE].

[21] M.S. Chanowitz, Strong WW scattering at the end of the 90's: theory and experimental prospects, hep-ph/9812215 [INSPIRE].

[22] S. Dawson, The effective W approximation, Nucl. Phys. B 249 (1985) 42 [INSPIRE].

[23] G.L. Kane, W.W. Repko and W.B. Rolnick, The effective $W^{ \pm}, Z^{0}$ approximation for high-energy collisions, Phys. Lett. B 148 (1984) 367 [INSPIRE].

[24] J. Lindfors, Distribution functions for heavy vector bosons inside colliding particle beams, Z. Phys. C 28 (1985) 427 [INSPIRE].

[25] M.S. Chanowitz and M.K. Gaillard, Multiple production of $W$ and $Z$ as a signal of new strong interactions, Phys. Lett. B 142 (1984) 85 [INSPIRE].

[26] J.F. Gunion, J. Kalinowski and A. Tofighi-Niaki, Exact $f f \rightarrow f f W W$ calculation for the charged current sector and comparison with the effective $W$ approximation, Phys. Rev. Lett. 57 (1986) 2351 [INSPIRE].

[27] E. Accomando, A. Ballestrero, A. Belhouari and E. Maina, Isolating vector boson scattering at the LHC: gauge cancellations and the equivalent vector boson approximation vs. complete calculations, Phys. Rev. D 74 (2006) 073010 [hep-ph/0608019] [INSPIRE].

[28] A. Alboteanu, W. Kilian and J. Reuter, Resonances and unitarity in weak boson scattering at the LHC, JHEP 11 (2008) 010 [arXiv:0806.4145] [INSPIRE].

[29] W. Kilian, T. Ohl and J. Reuter, WHIZARD: simulating multi-particle processes at LHC and ILC, Eur. Phys. J. C 71 (2011) 1742 [arXiv:0708.4233] [INSPIRE].

[30] M. Moretti, T. Ohl and J. Reuter, O'Mega: an optimizing matrix element generator, hep-ph/0102195 [INSPIRE].

[31] J.R. Forshaw, Selected topics in rapidity gap physics, hep-ph/0212154 [INSPIRE].

[32] L. Randall, Two Higgs models for large $\tan \beta$ and heavy second Higgs, JHEP 02 (2008) 084 [arXiv:0711.4360] [INSPIRE].

[33] G.F. Giudice, C. Grojean, A. Pomarol and R. Rattazzi, The strongly-interacting light Higgs, JHEP 06 (2007) 045 [hep-ph/0703164] [INSPIRE].

[34] A. Falkowski, C. Grojean, A. Kaminska, S. Pokorski and A. Weiler, If no Higgs then what?, JHEP 11 (2011) 028 [arXiv:1108.1183] [InSPIRE].

[35] D. Elander and M. Piai, A composite light scalar, electro-weak symmetry breaking and the recent $L H C$ searches, arXiv:1112.2915 [INSPIRE].

[36] K. Cheung, C.-W. Chiang and T.-C. Yuan, Partially strong WW scattering, Phys. Rev. D 78 (2008) 051701 [arXiv:0803.2661] [InSPIRE].

[37] T. Han, D. Krohn, L.-T. Wang and W. Zhu, New physics signals in longitudinal gauge boson scattering at the LHC, JHEP 03 (2010) 082 [arXiv:0911.3656] [INSPIRE].

[38] S.D. Rindani, Strong gauge boson scattering at the LHC, arXiv:0910.5068 [INSPIRE]. 
[39] H. Georgi, A tool kit for builders of composite models, Nucl. Phys. B 266 (1986) 274 [INSPIRE].

[40] R. Casalbuoni, S. De Curtis, D. Dominici and R. Gatto, Effective weak interaction theory with possible new vector resonance from a strong Higgs sector, Phys. Lett. B 155 (1985) 95 [INSPIRE].

[41] R. Casalbuoni, S. De Curtis, D. Dominici and R. Gatto, Physical implications of possible $J=1$ bound states from strong Higgs, Nucl. Phys. B 282 (1987) 235 [inSPIRE].

[42] M. Bando, T. Kugo, S. Uehara, K. Yamawaki and T. Yanagida, Is rho meson a dynamical gauge boson of hidden local symmetry?, Phys. Rev. Lett. 54 (1985) 1215 [INSPIRE].

[43] H. Georgi, Vector realization of chiral symmetry, Nucl. Phys. B 331 (1990) 311 [InSPIRE].

[44] R. Casalbuoni, S. De Curtis, D. Dominici, F. Feruglio and R. Gatto, Vector and axial vector bound states from a strongly interacting electroweak sector, Int. J. Mod. Phys. A 4 (1989) 1065 [inSPIRE].

[45] C. Csáki, C. Grojean, H. Murayama, L. Pilo and J. Terning, Gauge theories on an interval: unitarity without a Higgs, Phys. Rev. D 69 (2004) 055006 [hep-ph/0305237] [INSPIRE].

[46] R. Foadi, S. Gopalakrishna and C. Schmidt, Higgsless electroweak symmetry breaking from theory space, JHEP 03 (2004) 042 [hep-ph/0312324] [INSPIRE].

[47] H. Georgi, Fun with Higgsless theories, Phys. Rev. D 71 (2005) 015016 [hep-ph/0408067] [INSPIRE].

[48] R.S. Chivukula, D.A. Dicus and H.-J. He, Unitarity of compactified five-dimensional Yang-Mills theory, Phys. Lett. B 525 (2002) 175 [hep-ph/0111016] [INSPIRE].

[49] R.S. Chivukula and H.-J. He, Unitarity of deconstructed five-dimensional Yang-Mills theory, Phys. Lett. B 532 (2002) 121 [hep-ph/0201164] [INSPIRE].

[50] R.S. Chivukula, D.A. Dicus, H.-J. He and S. Nandi, Unitarity of the higher dimensional standard model, Phys. Lett. B 562 (2003) 109 [hep-ph/0302263] [INSPIRE].

[51] H.-J. He, Higgsless deconstruction without boundary condition, Int. J. Mod. Phys. A 20 (2005) 3362 [hep-ph/0412113] [InSPIRE].

[52] C. Csáki, C. Grojean, J. Hubisz, Y. Shirman and J. Terning, Fermions on an interval: quark and lepton masses without a Higgs, Phys. Rev. D 70 (2004) 015012 [hep-ph/0310355] [INSPIRE].

[53] Y. Nomura, Higgsless theory of electroweak symmetry breaking from warped space, JHEP 11 (2003) 050 [hep-ph/0309189] [INSPIRE].

[54] M. Papucci, NDA and perturbativity in Higgsless models, hep-ph/0408058 [INSPIRE].

[55] A. Muck, L. Nilse, A. Pilaftsis and R. Ruckl, Quantization and high energy unitarity of 5-D orbifold theories with brane kinetic terms, Phys. Rev. D 71 (2005) 066004 [hep-ph/0411258] [INSPIRE].

[56] A. Falkowski, S. Pokorski and J.P. Roberts, Modelling strong interactions and longitudinally polarized vector boson scattering, JHEP 12 (2007) 063 [arXiv:0705.4653] [INSPIRE].

[57] R. Barbieri, G. Isidori, V.S. Rychkov and E. Trincherini, Heavy vectors in Higgs-less models, Phys. Rev. D 78 (2008) 036012 [arXiv:0806.1624] [INSPIRE]. 
[58] N. Arkani-Hamed, A.G. Cohen and H. Georgi, (De)constructing dimensions, Phys. Rev. Lett. 86 (2001) 4757 [hep-th/0104005] [INSPIRE].

[59] N. Arkani-Hamed, A.G. Cohen and H. Georgi, Electroweak symmetry breaking from dimensional deconstruction, Phys. Lett. B 513 (2001) 232 [hep-ph/0105239] [INSPIRE].

[60] C.T. Hill, S. Pokorski and J. Wang, Gauge invariant effective Lagrangian for Kaluza-Klein modes, Phys. Rev. D 64 (2001) 105005 [hep-th/0104035] [INSPIRE].

[61] H.-C. Cheng, C.T. Hill, S. Pokorski and J. Wang, The standard model in the latticized bulk, Phys. Rev. D 64 (2001) 065007 [hep-th/0104179] [INSPIRE].

[62] H. Abe, T. Kobayashi, N. Maru and K. Yoshioka, Field localization in warped gauge theories, Phys. Rev. D 67 (2003) 045019 [hep-ph/0205344] [INSPIRE].

[63] A. Falkowski and H.D. Kim, Running of gauge couplings in AdS $S_{5}$ via deconstruction, JHEP 08 (2002) 052 [hep-ph/0208058] [INSPIRE].

[64] L. Randall, Y. Shadmi and N. Weiner, Deconstruction and gauge theories in AdS $S_{5}$, JHEP 01 (2003) 055 [hep-th/0208120] [INSPIRE].

[65] D.T. Son and M.A. Stephanov, $Q C D$ and dimensional deconstruction, Phys. Rev. D 69 (2004) 065020 [hep-ph/0304182] [INSPIRE].

[66] J. de Blas, A. Falkowski, M. Pérez-Victoria and S. Pokorski, Tools for deconstructing gauge theories in $A d S_{5}, J H E P 08$ (2006) 061 [hep-th/0605150] [INSPIRE].

[67] J. Hirn and J. Stern, The role of spurions in Higgsless electroweak effective theories, Eur. Phys. J. C 34 (2004) 447 [hep-ph/0401032] [INSPIRE].

[68] R. Casalbuoni, S. De Curtis and D. Dominici, Moose models with vanishing S parameter, Phys. Rev. D 70 (2004) 055010 [hep-ph/0405188] [inSPIRE].

[69] R.S. Chivukula, E.H. Simmons, H.-J. He, M. Kurachi and M. Tanabashi, The structure of corrections to electroweak interactions in Higgsless models, Phys. Rev. D 70 (2004) 075008 [hep-ph/0406077] [INSPIRE].

[70] R.S. Chivukula, E.H. Simmons, H.-J. He, M. Kurachi and M. Tanabashi, Universal non-oblique corrections in Higgsless models and beyond, Phys. Lett. B 603 (2004) 210 [hep-ph/0408262] [INSPIRE].

[71] M. Perelstein, Gauge-assisted technicolor?, JHEP 10 (2004) 010 [hep-ph/0408072] [INSPIRE].

[72] R.S. Chivukula et al., A three site Higgsless model, Phys. Rev. D 74 (2006) 075011 [hep-ph/0607124] [INSPIRE].

[73] R. Foadi, M.T. Frandsen, T.A. Ryttov and F. Sannino, Minimal walking technicolor: set up for collider physics, Phys. Rev. D 76 (2007) 055005 [arXiv:0706.1696] [InSPIRE].

[74] R. Barbieri, A.E. Carcamo Hernandez, G. Corcella, R. Torre and E. Trincherini, Composite vectors at the Large Hadron Collider, JHEP 03 (2010) 068 [arXiv:0911.1942] [INSPIRE].

[75] E. Accomando, S. De Curtis, D. Dominici and L. Fedeli, Drell-Yan production at the LHC in a four site Higgsless model, Phys. Rev. D 79 (2009) 055020 [arXiv:0807.5051] [INSPIRE].

[76] R. Barbieri, G. Isidori and D. Pappadopulo, Composite fermions in electroweak symmetry breaking, JHEP 02 (2009) 029 [arXiv:0811.2888] [INSPIRE]. 
[77] A.E. Carcamo Hernandez, Composite vectors and scalars in theories of electroweak symmetry breaking, arXiv:1108.0115 [INSPIRE].

[78] F. Bernardini, F. Coradeschi and D. Dominici, Effective chiral Lagrangians for new vector bosons to $O\left(p^{4}\right)$ order, arXiv:1111.4904 [INSPIRE].

[79] C. Csáki, J. Erlich and J. Terning, The effective Lagrangian in the Randall-Sundrum model and electroweak physics, Phys. Rev. D 66 (2002) 064021 [hep-ph/0203034] [INSPIRE].

[80] K. Agashe, A. Delgado, M.J. May and R. Sundrum, RS1, custodial isospin and precision tests, JHEP 08 (2003) 050 [hep-ph/0308036] [INSPIRE].

[81] The ILD Concept Group, The International Large Detector: letter of intent, arXiv:1006.3396 [INSPIRE].

[82] ILC collaboration, G. Aarons et al., International Linear Collider reference design report. Volume 2: Physics at the ILC, arXiv:0709.1893 [INSPIRE].

[83] ECFA/DESY LC Physics Working Group collaboration, J. Aguilar-Saavedra et al., TESLA: the superconducting electron positron linear collider with an integrated X-ray laser laboratory. Technical design report. Part 3: Physics at an $e^{+} e^{-}$linear collider, hep-ph/0106315 [INSPIRE].

[84] M. Beyer et al., Determination of new electroweak parameters at the ILC - sensitivity to new physics, Eur. Phys. J. C 48 (2006) 353 [hep-ph/0604048] [INSPIRE].

[85] T.L. Barklow et al., Electroweak symmetry breaking by strong dynamics and the collider phenomenology, in Proceedings of the APS/DPF/DPB Summer Study on the Future of Particle Physics (Snowmass 2001), Snowmass U.S.A., 30 Jun-21 Jul 2001 [hep-ph/0201243] [INSPIRE].

[86] T.L. Barklow, Strong symmetry breaking at $e^{+} e^{-}$linear colliders, in Proceedings of the APS/DPF/DPB Summer Study on the Future of Particle Physics (Snowmass 2001), Snowmass U.S.A., 30 Jun-21 Jul 2001 [hep-ph/0112286] [INSPIRE].

[87] D. Dominici, Tests for a strong electroweak sector at future $e^{+} e^{-}$high-energy colliders, Riv. Nuovo Cim. 20 (1997) 1 [hep-ph/9711385] [INSPIRE].

[88] R. Casalbuoni et al., Vector resonances from a strong electroweak sector at linear colliders, Z. Phys. C 60 (1993) 315 [hep-ph/9303201] [INSPIRE].

[89] R. Casalbuoni, S. De Curtis and D. Guetta, Study of the anomalous couplings at NLC with polarized beams, contributed to 5th Workshop of the 2nd ECFA-DESY Study on Physics and Detectors for a Linear Electron-Positron Collider, Obernai France, 16-19 Oct 1999, in 2nd ECFA/DESY Study 1998-2001, pp. 351-365 [hep-ph/9912377] [INSPIRE].

[90] P. Poulose, S.D. Rindani and L.M. Sehgal, Lepton spectra from $e^{+} e^{-} \rightarrow W^{+} W^{-}$in the BESS model, Phys. Lett. B 525 (2002) 71 [hep-ph/0111134] [INSPIRE].

[91] F. Iddir, A. Le Yaouanc, L. Oliver, O. Pene and J.C. Raynal, $W^{+} W^{-}$production in $e^{+} e^{-}$ colliders: a test of a strongly interacting Higgs sector, Phys. Rev. D $4 \mathbf{1}$ (1990) 22 [INSPIRE].

[92] A. Werthenbach and L.M. Sehgal, Energy spectrum of leptons from $e^{+} e^{-} \rightarrow W^{+} W^{-}$in the presence of strong $W_{L}^{+} W_{L}^{-}$interaction, Phys. Lett. B 402 (1997) 189 [hep-ph/9701255] [INSPIRE].

[93] W. Bernreuther and T. Schroder, Strongly interacting Higgs sector and $W$ pair production in $e^{+} e^{-}$collisions, Z. Phys. C 62 (1994) 615 [INSPIRE]. 
[94] P. Poulose and L.M. Sehgal, Strong final state interactions in $\gamma \gamma \rightarrow W W$, Phys. Lett. B 552 (2003) 57 [hep-ph/0211179] [INSPIRE].

[95] B. Ananthanarayan, M. Patra and P. Poulose, Signals of additional $Z$ boson in $e^{+} e^{-} \rightarrow W^{+} W^{-}$at the ILC with polarized beams, JHEP 02 (2011) 043 [arXiv:1012.3566] [INSPIRE].

[96] P. Langacker, The physics of heavy Z' gauge bosons, Rev. Mod. Phys. 81 (2009) 1199 [arXiv:0801.1345] [INSPIRE].

[97] B. Ananthanarayan, M. Patra and P. Poulose, $W$ physics at the ILC with polarized beams as a probe of the littlest Higgs model, JHEP 11 (2009) 058 [arXiv: 0909.5323] [INSPIRE].

[98] G. Moortgat-Pick et al., The role of polarized positrons and electrons in revealing fundamental interactions at the linear collider, Phys. Rept. 460 (2008) 131 [hep-ph/0507011] [INSPIRE].

[99] M. Diehl, O. Nachtmann and F. Nagel, Triple gauge couplings in polarized $e^{-} e^{+} \rightarrow W^{-} W^{+}$ and their measurement using optimal observables, Eur. Phys. J. C 27 (2003) 375 [hep-ph/0209229] [INSPIRE].

[100] M. Diehl, O. Nachtmann and F. Nagel, Probing triple gauge couplings with transverse beam polarisation in $e^{+} e^{-} \rightarrow W^{+} W^{-}$, Eur. Phys. J. C 32 (2003) 17 [hep-ph/0306247] [INSPIRE].

[101] F. Franco-Sollova, A study of triple gauge boson couplings in W-pair production at the linear collider using transversely polarized beams, LC-PHSM-2004-011 (2004) [INSPIRE].

[102] J. Fleischer, K. Kolodziej and F. Jegerlehner, Transverse versus longitudinal polarization effects in $e^{+} e^{-} \rightarrow W^{+} W^{-}$, Phys. Rev. D 49 (1994) 2174 [inSPIRE].

[103] T.N. Truong, Remarks on the unitarization methods, Phys. Rev. Lett. 67 (1991) 2260 [INSPIRE].

[104] K. Hagiwara, R.D. Peccei, D. Zeppenfeld and K. Hikasa, Probing the weak boson sector in $e^{+} e^{-} \rightarrow W^{+} W^{-}$, Nucl. Phys. B 282 (1987) 253 [INSPIRE].

[105] K.-i. Hikasa, Transverse polarization effects in $e^{+} e^{-}$collisions: the role of chiral symmetry, Phys. Rev. D 33 (1986) 3203 [inSPIRE].

[106] K.-i. Hikasa, Strongly interacting Higgs sector, in Proceedings of the Workshop on Physics with Linear Colliders, Saariselkae Finland, 9-14 Sep 1991, Vol. 2, pp. 451-487, KEK Preprint KEK-TH-319 (1992) [INSPIRE].

[107] D.A. Dicus and K. Kallianpur, Energy distribution of leptons from $e^{+} e^{-} \rightarrow W^{+} W^{-}$, Phys. Rev. D 32 (1985) 35 [inSPIRE]. 